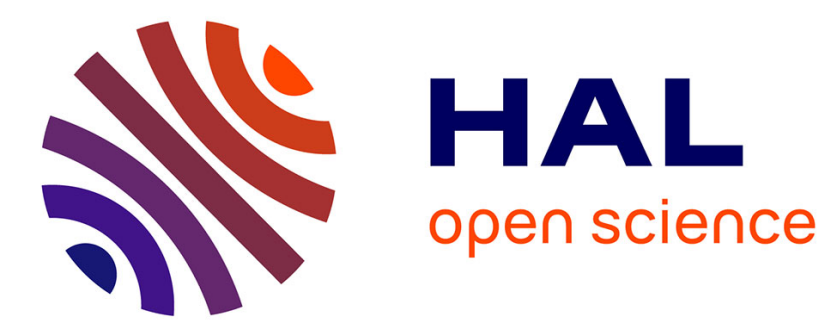

\title{
Numerical Analysis of Porous Coatings Stabilizing Capabilities on Hypersonic Boundary-Layer Transition
}

Romain Fiévet, Hugues Deniau, Jean-Philippe Brazier, Estelle Piot

\section{To cite this version:}

Romain Fiévet, Hugues Deniau, Jean-Philippe Brazier, Estelle Piot. Numerical Analysis of Porous Coatings Stabilizing Capabilities on Hypersonic Boundary-Layer Transition. AIAA Journal, 2021, 59 (10), pp.3845-3858. 10.2514/1.J059830 . hal-03280175

\section{HAL Id: hal-03280175 https://hal.science/hal-03280175}

Submitted on 7 Jul 2021

HAL is a multi-disciplinary open access archive for the deposit and dissemination of scientific research documents, whether they are published or not. The documents may come from teaching and research institutions in France or abroad, or from public or private research centers.
L'archive ouverte pluridisciplinaire HAL, est destinée au dépôt et à la diffusion de documents scientifiques de niveau recherche, publiés ou non, émanant des établissements d'enseignement et de recherche français ou étrangers, des laboratoires publics ou privés. 


\title{
Numerical analysis of porous coatings stabilizing capabilities on hypersonic boundary-layer transition
}

\author{
Romain Fiévet *, Hugues Deniau ${ }^{\dagger}$, Jean-Philippe Brazier ${ }^{\ddagger}$ and Estelle Piot $^{\S}$ \\ ONERA, University of Toulouse, F-31055, Toulouse, France
}

\begin{abstract}
A high-order spectral difference flow solver is used to perform direct numerical simulations (DNS) of a hypersonic laminar boundary layer on an ultrasonically absorptive coating (UAC), in order to analyze the stabilizing effects of such a material on the laminar-turbulent transition. The UAC is modeled in the simulations by a time-domain impedance boundary condition, which is calibrated to replicate the acoustic response of the UAC over a broad spectral range. The frequency-dependence damping effectiveness is investigated, as well as any other side-effects the porous might have on the mean flow. It is found that the second-mode instability, which dominates high-Mach number flow regime, is strongly suppressed for all excitation frequencies considered. The DNS solutions are also found to compare favorably with linearized stability theory. Finally, a side effect of the UAC is the modification of the medium dispersive properties and the change of the perturbation group velocity.
\end{abstract}

\section{Nomenclature}

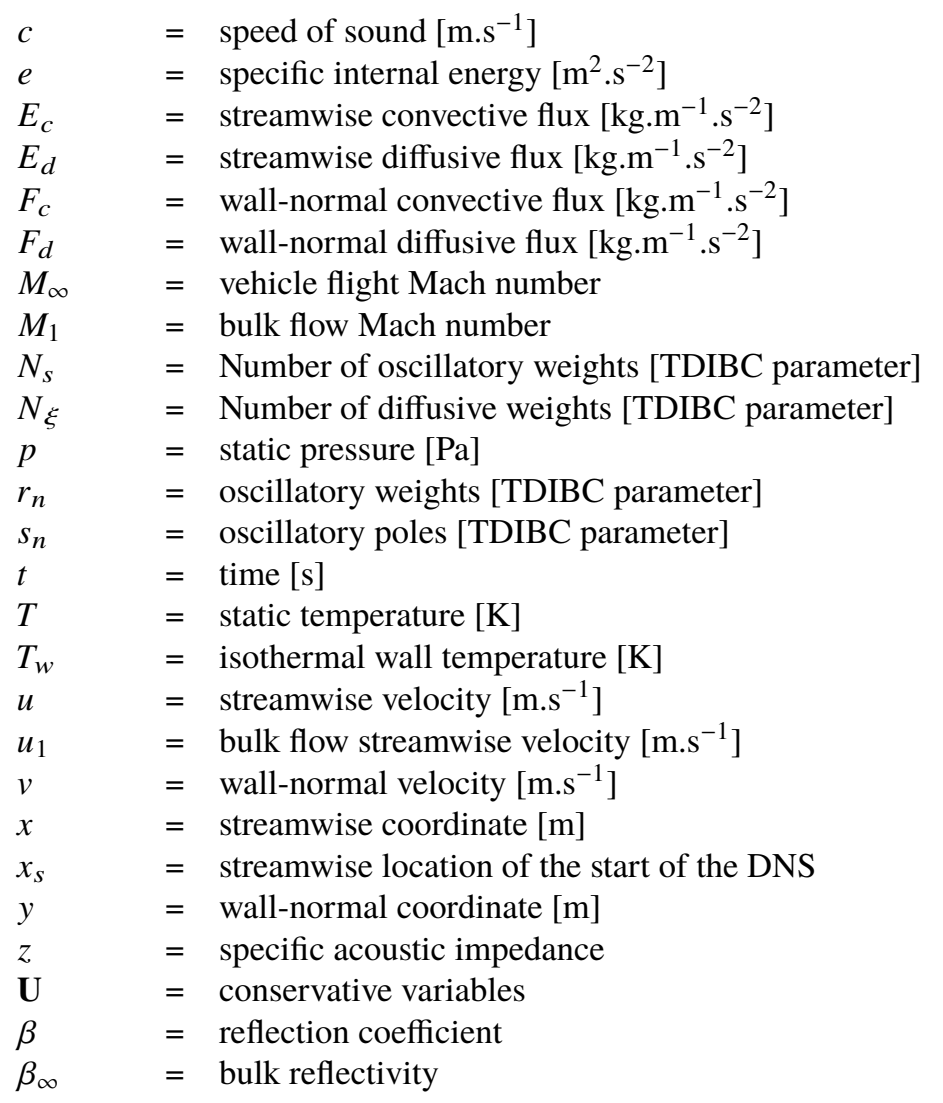

\footnotetext{
*Postdoctoral Researcher, currently at the Niels Bohr Institute, University of Copenhagen, fievetromain@ gmail.com

$\dagger$ Research Engineer, Department of Multi-Physics and Energetics, hugues.deniau@onera.fr

†Research Engineer, Department of Multi-Physics and Energetics, jean-philippe.brazier@onera.fr

${ }^{\S}$ Research Engineer, Department of Multi-Physics and Energetics, estelle.piot@onera.frOrlando, FL, AIAA 2020-2061
} 


$\begin{array}{lll}\eta & = & \text { second generalized coordinate } \\ \lambda_{T} & = & \text { thermal conductivity }\left[\mathrm{J} \cdot \mathrm{K}^{-1} \cdot \mathrm{s}^{-1}\right] \\ \mu_{k} & = & \text { diffusive weights }[\mathrm{TDIBC} \text { parameter] } \\ \rho & = & \text { flow density }\left[\mathrm{kg} \cdot \mathrm{m}^{-3}\right] \\ \left.\sigma_{c}\right] & =\text { Wall porosity } \\ \tau & = & \text { time-delay }[\mathrm{TDIBC} \text { parameter] } \\ \tau_{(i, j)} & =\text { viscous stress tensor }\left[\mathrm{kg} \cdot \mathrm{m}^{-1} \cdot \mathrm{s}^{-2}\right] \\ \xi & = & \text { first generalized coordinate } \\ \xi_{k} & = & \text { diffusive poles [TDIBC parameter] } \\ \text { Superscripts } & \\ - & =\text { Time-averaged quantity } \\ , & =\text { Departure from average } \\ * & =\text { Quantity normalized by maximum value } \\ \text { Acronyms } & \\ \text { DNS } & =\text { Direct Numerical Simulation } \\ \text { LST } & =\text { Linear Stability Theory } \\ \text { NSCBC } & =\text { Navier-Stokes Characteristic Boundary Conditions } \\ \text { HBL } & =\text { Hypersonic Boundary Layer } \\ \text { ODE } & =\text { Ordinary Differential Equation } \\ \text { ODR } & =\text { Oscillatory Diffusive Representation } \\ \text { SD } & =\text { Spectral Difference } \\ \text { SSAS } & =\text { Single-Sided Amplitude Spectra } \\ \text { TDIBC } & =\text { Time-Domain Impedance Boundary Condition } \\ \text { UAC } & =\text { Ultrasonically Absorptive Coatings } \\ \text { 1D } & =\text { One-dimensional } \\ \text { 2D } & =\text { Two-dimensional }\end{array}$

\section{Introduction}

One of the great challenges faced in hypersonic vehicle design lies in tackling with the strong aerodynamic and thermal loads suffered during atmospheric flights. Both processes have a strong impact on the vehicle thrust-to-weight ratio and are, therefore, key design considerations. Coincidentally, they are known to be mitigated when the boundary layer remains laminar and exacerbated once it has become turbulent [1-3]. As a result, the laminar-turbulent transition of hypersonic boundary layers has been the focus of a large body of studies in the past decades, shedding light on the underlying physical mechanisms governing it.

In absence of bypass forcing, as is common assumption for high-altitude high-speed flights, transition is caused by the spatial growth of unstable modes [4]. At supersonic speeds, compressible Linear Stability Theory (LST) predicts the existence of an infinite number of modes [5], amongst which some are unstable (i.e. have a positive spatial growth-rate). The first unstable mode is the hypersonic equivalent of Tollmien-Schlichting waves and is predominant below Mach 4. The subsequent unstable modes, derived from the compressible inviscid Euler equations, are of acoustic nature and typically dominate the laminar-turbulent transition process beyond Mach 4 [5]. Such instabilities have been experimentally [3, 6, 7] and numerically [2, 8,-12] visualized as rope-like density waves wrapped around the critical layer in close vicinity to the wall. The acoustic analogy is further supported by the work of Kuehl [13] detailing how the wall-normal gradient of the acoustic impedance would form an impedance well which physically acts as an acoustic wave-guide inside the boundary layer. The resonating acoustic modes trapped inside it grow as they propagate along the streamwise direction. As they travel downstream, energy is progressively transferred from the mean laminar kinetic energy into unsteady acoustic energy until turbulence erupts [13]. Interestingly, wall cooling further destabilizes the higher modes contrary to the first viscous mode [5, 14] as it exacerbates wall-normal thermal gradient and, therefore, strengthen the impedance well. These unstable vortico-acoustic modes are ordered by increased frequency and the second one typically dominates in the low-hypersonic regime [5]. Hence, it is of critical interest to design second-mode stabilizing systems to delay the laminar-to-turbulent transition in HBL.

One of the most promising stabilizing scheme consists in covering the vehicle with a porous coating. The pores act as acoustic resonators which are designed to filter out specific frequencies. It was theorized by Fedorov in 2001 [15] and experimentally validated by Rasheed $e t$ al. the following year [16] in a Mach 5 conical flow configuration. These 
seminal studies have, since then, encouraged the development of Ultrasonically Absorptive Coating (UAC) designed to absorb second-mode waves [17--21]. Importantly, it was observed that the wall porosity has a broadband effect on the acoustic field: that is, it could coincidentally destabilize the first [8, 17] and higher modes [22] while stabilizing the second mode. Hence, the coating's acoustic properties must be carefully tuned to respectively maximize/minimize the spectral canceling/reinforcement regimes. Given the manufacturing cost each experimental trial would involve, a numerical approach is appealing for the design of an optimal UAC.

Computational studies have long proven their ability to capture the UAC stabilizing effect by either directly resolving the flow inside the porous coating [22-26] or by modeling its impedance as a boundary condition [27--31]. The direct numerical simulation (DNS) of Brès et al. [22] resolved the UAC damping effects using the former method by meshing the pores as uniformly-spaced cavities. This provided important insight on pore-to-pore interactions and revealed the presence of a slip-velocity induced instability in the high-porosity shallow-pores limit. However, the computational cost limited the scale of the simulation over a single second-mode wavelength, focusing on temporal rather than the more relevant spatial instabilities. To circumvent the need to mesh the porosity, time-domain impedance boundary conditions (TDIBC) mimicking the UAC response over a delimited spectral range have been successfully used in linear [27, 28, 31] and nonlinear solvers alike [29, 30]. Of particular relevance to the current study is the latter work which performed a DNS aimed at capturing the whole transition region with TDIBC calibrated from experimental measurements of UAC impedance.

Recent progresses in the development of TDIBC for high-order methods include the work of Monteghetti et al. [32, 33] and Fiévet et al. [34]. First, Monteghetti et al. derived a formulation based on the Oscillatory Diffusive Representation (ODR). It allows to recast an arbitrarily-complex impedance law into a finite set of weighted acoustic poles which can easily be tuned to provide a broadband-accurate discrete approximation. This representation was used to derive a time-domain scattering operator which served as a TDIBC by coupling it with the linearized Euler equations. Importantly, this scattering formulation was demonstrated to offer better numerical stability and reduced timestep constraints in grazing flow configuration than an impedance-based one [32]. These properties are highly relevant to CFD applications. Latter, Fiévet et al. built on this work to adapt this ODR-based TDIBC to the Navier-Stokes equations by developing them as a subclass of the celebrated family of Navier-Stokes Characteristic Boundary Condition (NSCBC) [35]. Coincidentally, this last work was part of a larger research effort aimed at enabling NSCBC with strong discontinuous finite element methods, particularly with the Spectral Difference (SD) scheme. Such effort was motivated by the need to develop non-reflecting boundary conditions (a well-known subclass of NSCBC) with the SD method to enable its use in studying complex unsteady physics. Indeed, the SD method is highly-scalable and minimally-dissipative, both of which are necessary conditions to run large-scale DNS and resolve the broadband aero-acoustic interactions within the transition region. However, the use of such dissipative-less numerical method can prove counter-productive should spurious reflections occur upon interaction of outgoing waves with any boundary. The resulting back-traveling wave would contaminate the inner solution, potentially interacting with the physical phenomenon being scrutinized. In the case of laminar-turbulent transition, for instance, the physical growth of unstable modes could be affected by such numerical artifact. Hence, both non-reflecting and time-domain impedance boundary conditions were recently implemented with the novel SD method. The end result is a high-order numerical flow solver which has demonstrated ([34]) its ability to resolve acoustic liner flows in grazing incidence configurations with great accuracy on unstructured meshes.

With this background, the current project aims at studying the stabilizing capabilities of porous coatings on HBL by means of direct numerical simulation (DNS) using this state-of-the-art numerical flow solver. Importantly, this work develops that presented in [36]. The configuration mimics the experiment of Wagner et al. [19] which corresponds to a two-dimensional (2D) $7^{\circ}$-angled dihedral with $27 \mathrm{~km}$-altitude Mach 7.4 flight far-field conditions. In their experiment, a UAC of their design successfully stabilized the HBL. The porous coating's acoustic properties were measured in Sousa et al. [29] and can be used to accurately calibrate the ODR-based TDIBC over a broad spectral range. Its response to harmonic/broadband forced excitation will then be scrutinized. LST will be used to assess the accuracy of the DNS. Importantly, this study is also meant to serve as a milestone as inverse-problem solving methods are being developed within the solver by means of automatic differentiation algorithms [37]. They will be used in the near-future to optimize the stabilizing capabilities of UAC as a function of flight conditions.

The paper is organized as follows. A brief overview of the numerical methods employed throughout this study is presented in a first section, with a description of the numerical experiment configuration and protocol. The next section presents the simulations and analysis in the following order. First and foremost, the steady-state, unperturbed flow field is resolved with and without coating to verify the passivity condition of the TDIBC. Then, the flow is perturbed using a variety of source frequency and type in simulations which either use a UAC or a solid wall condition. The latter cases 
are meant to serve as reference simulations and verify that the DNS and LST predictions are coherent with one another. The former simulation quantifies the effect of the UAC on the perturbation and mean flow fields. Conclusions and acknowledgements respectively follow.

\section{Numerical methods and flow configuration}

A description of the conservation equations, numerical schemes and time-domain impedance boundary conditions used in the flow solver is given in this section. Then, the protocol of the numerical experiments constituting this study is detailed. Finally, the geometric configuration is designed in light of a linear stability analysis performed over a precursor simulation.

\section{A. Numerical flow solver and schemes}

The main numerical flow solver used in this project belong to the project JAGUAR (project of an Aerodynamic solver using $G$ eneral $U$ nstructured grids $A$ nd high orde $R$ schemes) and is actively co-developed by ONERA and CERFACS (European Center of Research and Formation in Advanced Scientific Calculus). It employs a spectral difference finite element scheme coupled with an explicit six-stage Runge-Kutta time-marching scheme. The order of the SD scheme can be changed by simply increasing the order of the flux reconstruction polynomial, as in other discontinuous finite element methods. All SD simulations presented in this study use a $4^{t h}$-order SD scheme. Importantly, Vanharen $e t$ al. [38] assessed the accuracy of the SD method for various orders and demonstrated its favorable comparison with spectral-like resolution methods such as the compact finite difference scheme [39, 40]. A spectral-like resolution implies that the numerical scheme causes minimal dissipation and dispersion errors. This is crucial when studying physical phenomenon where a broad range of wavenumbers need to be properly resolved, as in laminar-turbulent transition. Benchmarking and validation test cases using JAGUAR are available in [41, 42].

The conservation equations resolved in this study are the non-reacting Navier-Stokes equations in two-dimensional (2D) generalized coordinates $(\xi, \eta)$, which can be written as:

$$
\begin{aligned}
\frac{1}{J} \frac{\partial \mathbf{U}}{\partial t} & +\frac{\partial}{\partial \xi}\left(\mathbf{E}_{c} \frac{\xi_{x}}{J}+\mathbf{F}_{c} \frac{\xi_{y}}{J}\right)+\frac{\partial}{\partial \xi}\left(\mathbf{E}_{d} \frac{\xi_{x}}{J}+\mathbf{F}_{d} \frac{\xi_{y}}{J}\right) \\
& +\frac{\partial}{\partial \eta}\left(\mathbf{E}_{c} \frac{\eta_{x}}{J}+\mathbf{F}_{c} \frac{\eta_{y}}{J}\right)+\frac{\partial}{\partial \eta}\left(\mathbf{E}_{d} \frac{\eta_{x}}{J}+\mathbf{F}_{d} \frac{\eta_{y}}{J}\right)=0,
\end{aligned}
$$

where the convective and diffusive fluxes of $\mathbf{U}$ in physical space are respectively called $\left(\mathbf{E}_{c}, \mathbf{F}_{c}\right)$ and $\left(\mathbf{E}_{d}, \mathbf{F}_{d}\right)$. They read as:

$$
\begin{aligned}
& \mathbf{E}_{c}=\left(\rho u, \rho u^{2}+p, \rho v u,(\rho e+p) u\right)^{\top} \\
& \mathbf{F}_{c}=\left(\rho v, \rho u v, \rho v^{2}+p,(\rho e+p) v\right)^{\top} \\
& \mathbf{E}_{d}=\left(0, p+\tau_{(1,1)}, \tau_{(2,1)}, u \tau_{(1,1)}+v \tau_{(2,1)}+\lambda_{T} \partial_{x} T\right)^{\top} \\
& \mathbf{F}_{d}=\left(0, \tau_{(1,2)}, p+\tau_{(2,2)}, u \tau_{(1,2)}+v \tau_{(2,2)}+\lambda_{T} \partial_{y} T\right)^{\top}
\end{aligned}
$$

The flow dynamic viscosity is evaluated at each sub-iteration using Sutherland's law, and the thermal diffusivity is evaluated assuming a constant Prandtl number of 0.72. The air is considered at thermal equilibrium throughout the study and a $7^{\text {th }}$-order temperature polynomial is used to evaluate the heat capacity at constant pressure. Further details on the flow solver can be found in [34].

\section{B. Time-domain impedance boundary condition}

The porous coating of interest considered throughout this study is the ultrasonically absorptive carbon fiber reinforced carbon developed by the German Aerospace Laboratory [19, 20]. This UAC was successfully used to delay the laminar-to-turbulent transition on a conical surface in the experimental campaign mentioned in the introduction. It was carried out at the High-Enthalpy Goettingen facility and replicated the flow conditions of an high-enthalpy Mach 7.4 flight. The real and imaginary part of the UAC's complex specific impedance in the frequency-domain are 
given in [29]. The HBL configuration considered herein is designed to match as closely as possible the conditions of these aforementioned studies, starting with the derivation of a TDIBC mimicking a real UAC. This class of boundary condition is comprised of three elements. First, an impedance model representing the physical properties of the coating of interest is determined. Second, the said model is discretized by ODR and subsequently converted into a time-domain operator. This amounts to evaluating a convolution product. Last, a semi-discrete formulation of the TDIBC is needed to couple it with the numerical flow solver. A brief presentation of these steps is provided below, the interested reader being referred to [33] for a thorough derivation of a TDIBC and [34] for its application with an SD flow solver.

\section{Impedance modeling}

First, a physical model of the coating impedance $\hat{z}$ is chosen. It relates the wall pressure perturbations with the normal velocity fluctuations in the Laplace-domain as $\hat{p}^{\prime}=\hat{z} \hat{v}^{\prime}$ where a hat marks a complex quantity function of the Laplace variable $s=\mathrm{j} \omega$. In the current study, the reinforced carbon fiber coating is modeled as a single-degree of freedom acoustic liner which consists of a set of narrow cylindrical cavities of diameter $d_{c}$ and length $l_{c}$ with a rigid end, filled with air at thermodynamic state $(\rho, c)$ inside which acoustic waves propagate and dissipate. Each of these cavities physically act as an acoustic resonator. Using the $1 \mathrm{D}$ wave equation for a wavenumber $k_{c}(s)$ to model the cavities impedance leads to:

$$
\hat{z}(s)=\frac{\rho c}{\sigma_{c}} \operatorname{coth}\left(\mathrm{j} k_{c}(s) l_{c}\right) .
$$

The corresponding reflection coefficient $\beta$ is defined as:

$$
\hat{\beta}=\frac{\hat{z}-\rho c}{\hat{z}+\rho c}
$$

and relates the pressure-velocity perturbations as $\left[\hat{p}^{\prime}+(\rho c) \hat{v}^{\prime}\right]=\beta\left[\hat{p}^{\prime}-(\rho c) \hat{v}^{\prime}\right]$. Importantly, its module is always inferior or equal to unity which offers interesting stability properties to a $\beta$-based TDIBC as opposed to a $z$-based one, as shown in [33].

\section{Delayed multi-polar discretization}

The second step consists in approximating the physical impedance/reflection model into a more manageable discrete form by the means of an ODR. The reader is referred to Sec. 3.2 of [33] for a detailed derivation of the ODR of this particular physical model for single-degree of freedom acoustic liner. Essentially, it amounts a decomposition into an infinite sum of weighted direct and delayed acoustic pole functions. It reads as:

$$
\hat{\beta}(s)=\beta_{\infty}+\sum_{n \in I} \frac{r_{1, n}}{s-s_{n}}+\int_{0}^{\infty} \frac{\mu_{1}(\xi)}{s+\xi} \mathrm{d} \xi+e^{-s \tau}\left(\sum_{n \in I} \frac{r_{2, n}}{s-s_{n}}+\int_{0}^{\infty} \frac{\mu_{2}(\xi)}{s+\xi} \mathrm{d} \xi\right) .
$$

where the complex oscillatory poles $s_{n}$ are the zeros of $\hat{z}+\rho c$ and the diffusive poles $\xi$ are arbitrarily chosen to span the spectral range of interest for a given configuration. The delay $\tau$ corresponds to the back-and-forth wave traveling time inside the cavity. Finally, the weights $r_{n}$ and $\mu_{n}$ are evaluated to fit the analytical function (Eq. 5) following the routine thoroughly described in [33]. It is important to note that no approximation has yet been made: the model is exactly represented by an infinite sum of oscillatory and diffusive poles. The oscillatory poles form conjugate pairs which guarantees the bounded-reality of the associated scattering operator. Further, an approximated reflection coefficient can naturally be derived by only using a finite number of $N_{\xi}$ diffusive and $N_{s}$ oscillatory poles. Finally, this set of parameters can be optimized to match a target impedance law or experimental dataset. This final discrete and optimized form of the reflection coefficient is called $\tilde{\hat{\beta}}$ and reads as:

$$
\tilde{\hat{\beta}}(s)=\tilde{\beta}_{\infty}+\sum_{n=1}^{N_{s}} \frac{\tilde{r}_{1, n}}{s-\tilde{s}_{n}}+\sum_{k=1}^{N_{\xi}} \frac{\tilde{\mu}_{1, k}}{s+\tilde{\xi}_{k}}+e^{-s \tilde{\tau}}\left(\sum_{n=1}^{N_{s}} \frac{\tilde{r}_{2, n}}{s-\tilde{s}_{n}}+\sum_{k=1}^{N_{\xi}} \frac{\tilde{\mu}_{2, k}}{s+\tilde{\xi}_{k}}\right)
$$

where the quantities marked by a tilde are the result of the aforementioned optimization through linear regression as in [33]. In the current study, the ODR of the UAC of interest is performed using a large set of parameter to ensure that the resulting TDIBC accurately resolve the reflection coefficient over a broad spectral range encompassing the range resolved by the latter DNS. The UAC denoted as "optimized C/C-SiC" in [29] is here chosen, since this material is considered as the most realistic and promising for in flight transition delay. The acoustic properties of this carbon fiber 
coating were derived in [29] using an inverse Helmholtz solver. We use these profiles, after conversion of the complex impedance into the reflection coefficient through Eq. 4 to derive an optimized parameters set, given in the appendix in Tab. 2 yielding the reflection coefficient Bode diagrams shown in Fig. 11. Note that 4 diffusive poles and 8 oscillatory poles were used, with their respective conjugates which can simply be accounted for arithmetically as twice the real part of the oscillatory polar functions.

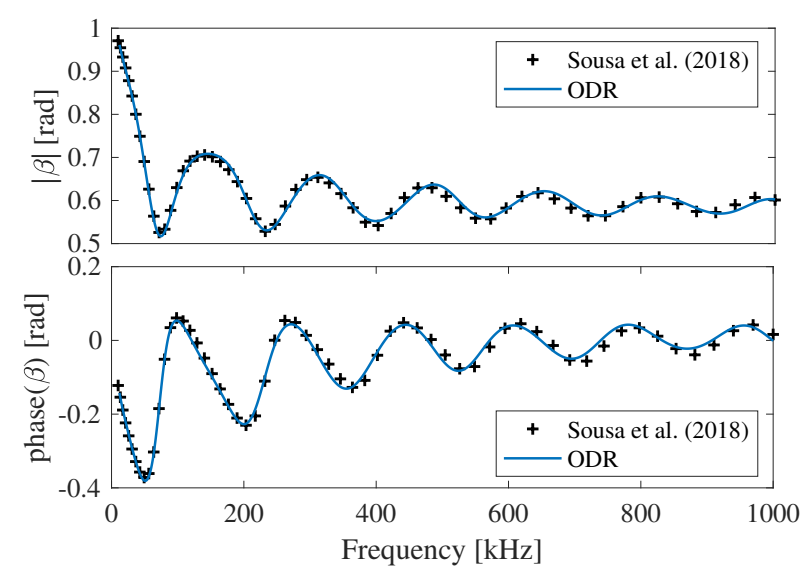

Fig. 1 (Top) Modulus and (Bottom) phase of the reflection coefficient of the UAC considered for this study as obtained in [29] by inverse Helmholtz analysis along its Oscillo-Diffusive Representation (ODR).

\section{Coupling with the Navier-Stokes equations}

Third, a time-domain scattering operator $\mathcal{B}$ is defined as:

$$
\partial_{t} p+(\rho c) \partial_{t} v=\mathcal{B}\left[\partial_{t} p-(\rho c) \partial_{t} v\right]=\tilde{\beta} \star\left[\partial_{t} p-(\rho c) \partial_{t} v\right]
$$

where $\mathcal{B}$ relates the rates of change of the reflected wave as a function of the incident wave. This amounts, as seen in Eq. 7. to a convolution product between the reflection coefficient inverse-Laplace transform, $\tilde{\beta}$, and the incident acoustic characteristic. The underlying interest of the ODR approach lies in the transformation of this convolution product into a sum of auxiliary functions as explained in [32]. Each auxiliary function amounts to a scalar contribution to the time-domain operator which corresponds to its associated polar function (Eq. (6)). The ODE are conveniently resolved using the same explicit time-marching scheme as the flow conservative variables $U$ to alleviate the computational cost of the TDIBC. $\mathcal{B}$ is then coupled with the Navier-Stokes equation as a characteristic boundary condition following the method presented in Sec. 3.2.2 and 3.2.3 in [34]. Specifically to the SD scheme, the TDIBC amounts to a compact (i.e. element-wise) flux balance correction applied in all the mesh's elements neighboring the UAC. The robustness of the implementation of an ODR-based scattering operator with the spectral difference scheme was extensively verified in [34] in grazing flow conditions, i.e. in a configuration similar to the present study.

\section{Presentation of the numerical experiment protocol}

The high-fidelity numerical methods presented above, integrated within the same flow solver, are used to simulate a hypersonic boundary-layer and study the UAC stabilization properties. To this end, an unperturbed, fully developed laminar boundary layer solution is first derived. It is then excited by the means of a wall blowing mechanism in order to trigger harmonic instabilities. Finally, the TDIBC is activated in order to study the UAC stabilizing capabilities and any other effect on the flow.

As explained in the introduction, the chosen configuration closely follows that of the experiment of Wagner et al. [19]. The same far-field conditions, corresponding to a $27 \mathrm{~km}$-altitude Mach $7.4 \mathrm{flight}$, are used. They are presented in Tab. 1. The model's geometry is chosen to be a $7^{\circ}$ half-angle $120 \mathrm{~cm}$-long two-dimensional dihedral instead of a cone, which constitutes the sole difference with the aforementioned experiment. While the properties of second-mode instabilities are known to differ from a cone to dihedral geometry, this design choice was motivated by computational cost reductions. As the goal of this study is not to perform a direct comparison with experimental data, this was deemed 


\begin{tabular}{|c|c|c|c|c|}
\hline \hline$R e_{x}\left[\mathrm{~m}^{-1}\right]$ & $M_{\infty}$ & $p_{\infty}[\mathrm{Pa}]$ & $T_{\infty}[\mathrm{K}]$ & $u_{\infty}[\mathrm{m} / \mathrm{s}]$ \\
\hline $4.06 \times 10^{6}$ & 7.4 & 2129 & 268 & 2422 \\
\hline
\end{tabular}

Table 1 Far-field conditions for the hypersonic dihedral simulation.

acceptable.

The numerical experiment protocol is now detailed. It comprises the following orderly steps:

1) A precursor solution of the dihedral flow is run with a high-enthalpy finite volume solver (code CEDRE [43, 44]) until time and grid convergence are reached. Its purpose is to serve as the unperturbed reference flows and initial state to subsequent runs. All rigid wall are treated as no-slip isothermal boundary condition with temperature $T_{w}=300 \mathrm{~K}$ for the remainder of the study. The configuration of this precursor simulation is presented in Fig. 2 The decision to rely on a different flow solver to perform the intake simulation was taken in light of the unavailability of robust shock-capturing techniques for the relatively novel SD scheme.

2) The wall-normal profiles are extracted from the steady precursor solution. An in-house linear stability theory (LST) solver (code MAMOUT) [45] is then used to determine the range of frequency of interest to be investigated, along with their associated growth region to be resolved by DNS.

3) The precursor flow field solution in the aforementioned region of interest is extracted. It is used to define the boundary conditions and initial state of the DNS to be run with the SD solver (code JAGUAR). A first unperturbed simulation is run to obtain a steady-state solution of a laminar HBL to be compared with the precursor solution. Importantly, this DNS uses characteristic boundary conditions at all domain edges which is, to the best of the authors knowledge, a novelty for a SD simulation of laminar-turbulent transition. Specifically, non-reflecting inflow/outflow and fully-reflective solid wall boundary conditions are used which ensures a proper treatment of perturbations at the domain's boundaries. The configuration for this and all subsequent runs correspond to the one presented in Fig. 3 where the wall blowing forcing and the TDIBC have been deactivated. The reasons behind this particular design are discussed in Sec. II.D.

4) Then, based on the LST results, the laminar HBL is excited at the frequencies $f$ identified in the second step by means of a permanent wall-blowing mechanism. Once a steady-state has been reached, the oscillation amplitudes are measured and the HBL response is compared with the LST. The SD code's capabilities in resolving the spatial growth rate of unstable modes are assessed by a direct comparison with the LST solution. The configuration for this run corresponds to the one presented in Fig. 3 where the TDIBC has been deactivated.

5) The TDIBC is then activated in the region of interest previously identified by the LST, and its stabilizing capabilities on the most unstable mode are assessed. Further, the effect of the UAC placement scheme on its stabilization properties are studied.

6) The steady-harmonic forcing is then replaced by a single wavepacket perturbation to observe the effect of broadband noise on the flow and the UAC.

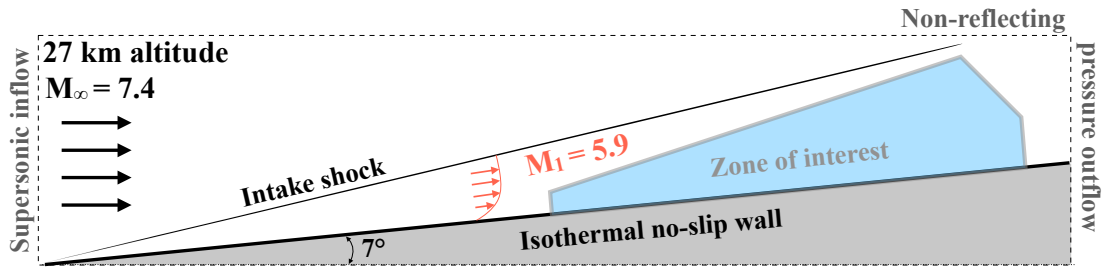

Fig. 2 Configuration of the hypersonic cone precursor simulation.

\section{Precursor simulation and linear stability analysis}

In accordance with Sec. II.C, the precursor simulation is first carried out to provide a mean flow solution over the dihedral (Fig. 2) which includes the intake shock. A 2D structured mesh was generated with a streamwise spatial increment $\delta x=1 \mu \mathrm{m}$ at the leading edge and a 1.01 growth factor. Likewise, the cells at the walls have a size of $\delta y=1$ 


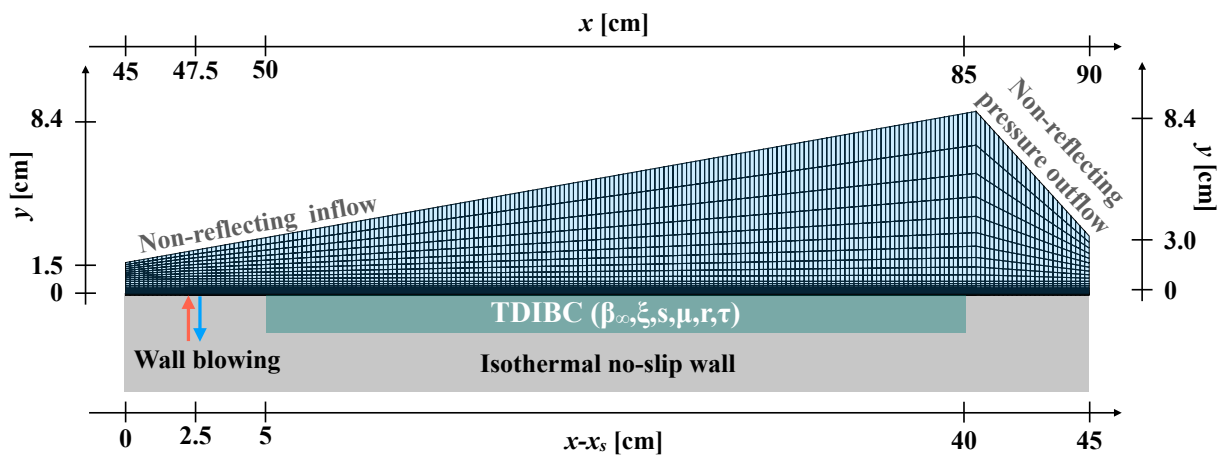

Fig. 3 Configuration of the perturbed hypersonic boundary layer DNS using the SD scheme, which corresponds to the "zone of interest" marked in Fig. 2. One one twenty-fourth mesh elements are shown.

$\mu \mathrm{m}$ and a 1.04 growth factor. As a result, the mesh is strongly clustered around the high-shear regions and is comprised of approximately 2.2 million elements. The boundary layer thickness is meshed with about 30 elements near the inlet, and this number increases along the cone, up to more than 100 elements at the outlet. The simulation is run using a first-order implicit time-integration scheme with $1 \mu$ s-long steps until a steady-state is reached as the maximum residue for density across the domain converged below $10^{-7}$. The fluxes are evaluated from a second-order flux reconstruction scheme using a HLLC Riemann solver[46] at the cell faces.

Once the solution has converged in time, the wall-normal velocity, temperature and pressure profiles at wall coordinate $x \in[20,120] \mathrm{cm}$ are extracted and fed into a LST solver. Notably, the flow behind the intake shock is a Mach 5.9 boundary layer with maximum streamwise velocity of $2300 \mathrm{~m} \cdot \mathrm{s}^{-1}$. The LST relies on the usual parallel-flow assumption. Further information on this code are provided in [45, 47]. The scope of the LST is to identify the growth region for specific disturbance frequencies, which remain to be determined. To this end, the experiment of Wagner $e t$ $a l$. [19] offers some precious insight: they identified a peak in wall pressure disturbance at around $260 \mathrm{kHz}$ at $60 \mathrm{~cm}$ from the leading edge (about half of the domain size shown in Fig. 2). Further, they observed that the most unstable modes, i.e. those with the highest spatial growth-rates, were roughly located between 180 and $300 \mathrm{kHz}$ in this region. Coincidentally, this corresponds to the target operating range of the UAC. In light of these experimental results, the flow stability is analyzed by the LST in the frequency range $[180,520] \mathrm{kHz}$. Especially, positive growth-rate regions are determined. These can be visualized by their corresponding $\mathrm{N}$-factor profiles along the streamwise direction. The $\mathrm{N}$-factor is defined as the natural logarithm of the pressure amplitude ratio from a reference position, or as the integral of the exponential spatial growth rate $-\alpha_{i}$ of the most unstable mode. These profiles are shown in Fig. 4. This dihedral flow has not exactly the same stability properties as the conical flow studied in the experiments, since the threshold value of 8 associated with natural transition was obtained then at approximately $75 \mathrm{~cm}$ [21]. Despite these differences in spatial growth rates, the unstable frequency band is indeed the same and corresponds to the UAC operating range. Frequencies lower than $180 \mathrm{kHz}$ are not expected to substantially destabilize the boundary layer within one meter of the leading edge. Frequencies higher than $420 \mathrm{kHz}$ will not cause a substantial growth anywhere in the HBL, with a maximum $\mathrm{N}$-factor below 4 located around $30 \mathrm{~cm}$ from the leading edge. The main frequency of interest to this study, $260 \mathrm{kHz}$, presents a positive growth-rate inside the $x \in[50,85] \mathrm{cm}$ region. Finally, the threshold value of 8 is reached by $120 \mathrm{~cm}$ for the $220 \mathrm{kHz}$ mode. As this study's interest lies in studying stabilization of laminar instabilities by UAC, the computational domain need not covering the late nonlinear transition process.

In light of these observations, it is decided to define the frequencies $f \in[180,240,260,280,300] \mathrm{kHz}$ as the modes to be investigated by the DNS. A special emphasize will be given on $260 \mathrm{kHz}$ which was singled-out as the most unstable one for these conditions. The region to be scrutinized with the SD solver is chosen to encompass the region $x \in[45,90] \mathrm{cm} . x_{s}=45 \mathrm{~cm}$ is defined as the starting streamwise location. The TDIBC is chosen to cover the 260 $\mathrm{kHz}$ growth region i.e. $x-x_{s} \in[5,40]$ and the wall-blowing forcing term is placed right upstream at $x-x_{s}=2.5$ $\mathrm{cm}$. As a result, this numerical experiment is tailored to study this mode in particular. A structured mesh comprising $N_{x} \times N_{y}=900 \times 180=162000$ orthogonal elements is used to discretize this domain. It is clustered around the wall with a smallest element size in the wall-normal direction $\delta y=10 \mu \mathrm{m}$ while a uniform streamwise spatial increment of $d x=0.5 \mathrm{~mm}$ is used. This amounts to a Nyquist frequency of $2 \mathrm{MHz}$ for a wave grazing over the wall at speed of $u_{\infty}-c$ which is the minimum propagation velocity of second-mode instabilities [3]. As the TDIBC is designed to resolve 


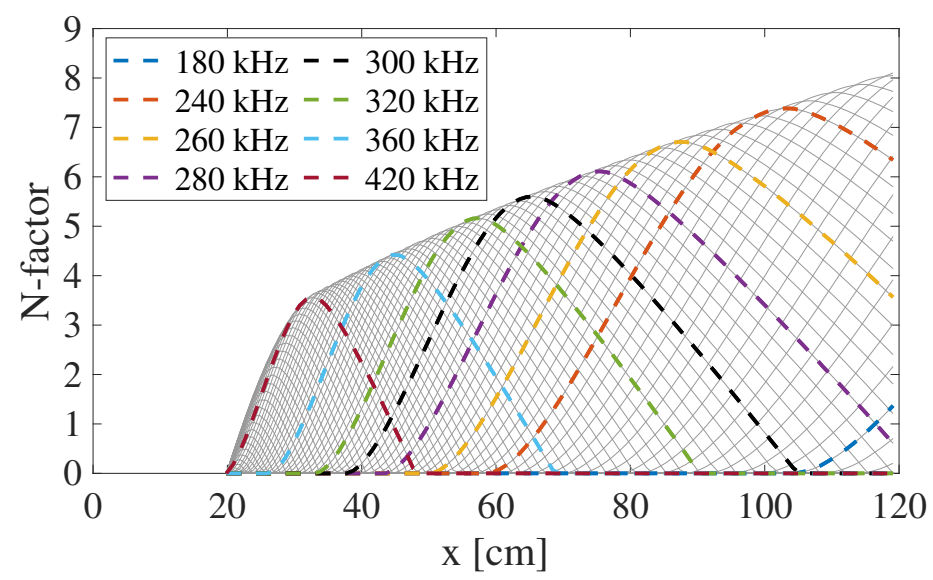

Fig. 4 N-factor profiles evaluated by the LST for frequencies from 180 to $520 \mathrm{kHz}$.

frequencies up to $500 \mathrm{kHz}$ and since the spectral range of interest is even more limited, the mesh resolution is deemed acceptable. Nonetheless, a grid convergence study will be carried out. At the inlet plane, i.e. $x=45 \mathrm{~cm}$, the boundary layer height $\delta$ (evaluated from the precursor simulation) equals $2.25 \mathrm{~mm}$. As a results, the DNS computational domain spans $200 \delta$ and has 84 cells across the boundary layer at its thinnest location. The sections of the inlet and outlet boundaries that are close to the wall are set normal to the streamwise direction, while they are angled further away from it. This allows to retain a projected hypersonic normal flow as much as possible in order to alleviate the computational cost at these boundaries. At supersonic outlets, the extrapolated solution from the inner domain is used to define the boundary condition. Conversely, supersonic inlets impose velocity, temperature and pressure fields extracted from the precursor solution. This concludes the motivations behind the design of the configuration shown in Fig. 3 which is the focus of the remainder of the study.

\section{Results and discussions}

Having designed and constrained the numerical experiment, various types of DNS are run by pairs with the SD code according to the protocol laid out in Sec. III.C Each of these pair includes one solid wall simulation and a second one where the TDIBC is activated. The first couple of runs simulate the unperturbed environment, i.e. a fully-laminar flow. The second batch of simulations uses a permanent harmonic wall-blowing perturbation to trigger instabilities and study their growth over both a solid wall and the UAC. The third and last set of simulations differs with the second one in that it uses a single wavepacket forcing function.

\section{A. Simulation of the initially unperturbed flow}

The precursor solution presented in Sec. [II.D is first interpolated onto the DNS domain. The resulting pressure, velocity and temperature fields both provide an initial state and extrapolated inflow/back-pressure boundary conditions at the computational domain's edges. A first solution of reference is run using solid wall conditions for over half a millisecond in physical time, using a Courant-Friedrich-Lewy number of 0.3 yielding a timestep of $4.5 \mathrm{~ns}$. This leaves enough time for any transient spurious waves triggered by the change of scheme and mesh to convect out of the domain. This operation is repeated while using the TDIBC mimicking the UAC presented in Fig. 1

Each of these runs used about 500 CPUh (5 hours on 100 computing cores) with the TDIBC run requiring an approximate $20 \%$ higher computational cost caused by the integration of auxiliary variables resolving the scattering operator. The wall-normal profiles of the streamwise and normal velocities ( $u$ and $v$, respectively) are extracted from both solution at several locations and plotted against the precursor solution in Figs. 5] and 6 . On the right hand-side of each figure, the normalized deviation relative to the solid wall DNS solution is plotted for the precursor solution and the UAC wall DNS solution, respectively. Strikingly, the velocity profiles are virtually indistinguishable between the solid and porous wall simulations. The highest absolute value of the normalized deviation between the UAC/solid wall cases is close to $0.01 \%$ which is largely within the bounds of accuracy of the DNS. The quasi-perfect match between these two solutions shows that the passivity of the wall boundary condition is verified. Passivity is a direct consequence of 
the TDIBC's well-posedness [32]. However, because of the change of mesh and scheme, the deviation between the precursor and DNS solutions can reach up to about $2 \%$ for the streamwise velocity and $20 \%$ for the wall-normal velocity. The wall-normal velocity deviation is the highest at the beginning of the DNS domain and decreases further downstream. This is attributed to the presence of a very weak pressure oblique discontinuity (on the order of 10 Pascal, i.e. $0.1 \%$ of the bulk static pressure) at the inlet plane, seemingly emanating from the sonic line. As a result, the DNS wall-pressure profiles (not shown for the sake of brevity) also present this small pressure defect with respect to the precursor solution. As the LST calculations rely on the parallel flow assumption, this small and unavoidable discrepancy has only little impact on the stability properties of each boundary layer. However, in order to get the best possible comparison with the DNS results, all the LST calculations will be based hereinafter on the DNS mean flow solution.
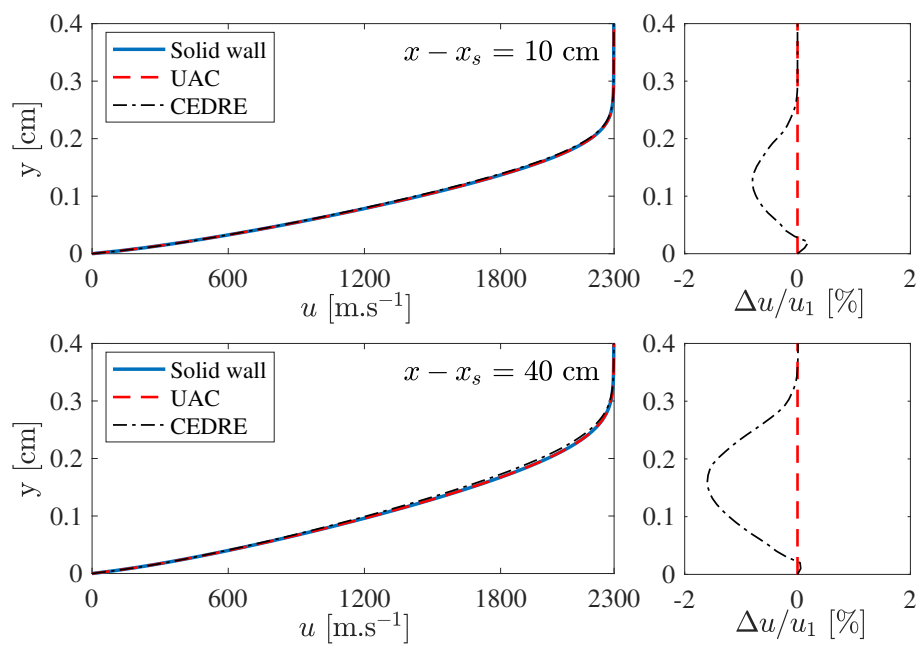

Fig. 5 Wall-normal profiles of streamwise velocity at different streamwise locations for the precursor (CEDRE) and laminar DNS solutions in solid wall or UAC configurations. $\Delta u$ is defined with respect to the solid wall DNS solution.
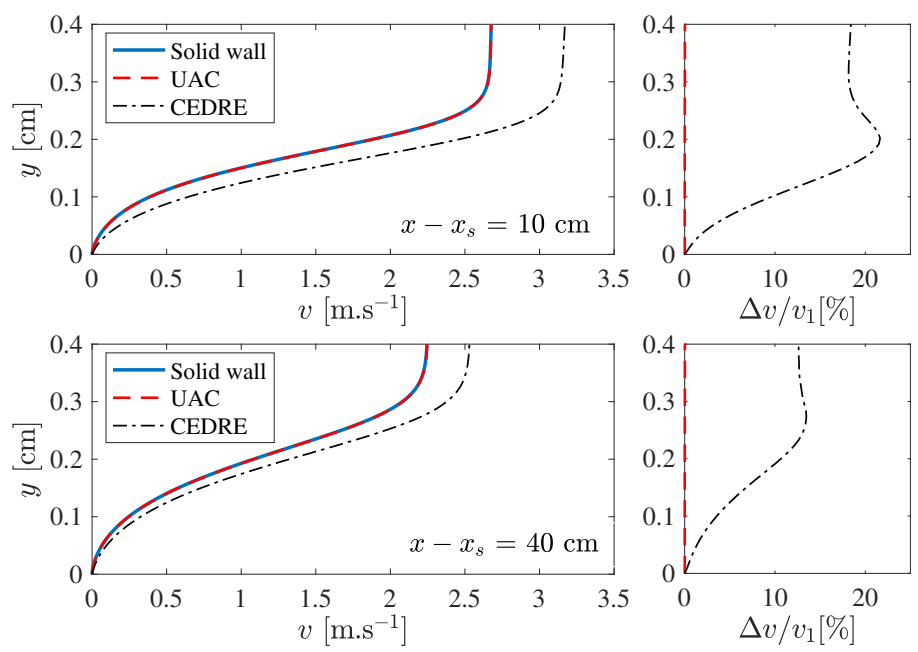

Fig. 6 Wall-normal profiles of wall-normal velocity at different streamwise locations for the precursor (CEDRE) and laminar DNS solutions in solid wall or UAC configurations. $\Delta v$ is defined with respect to the solid wall DNS solution.

Further, the steady and laminar solution of the solid wall case is used to visualize in Fig 7 the impedance well, as in 
[13], which is highlighted by the wall-normal gradient of the streamwise momentum. This area is growing along the streamwise direction, as the boundary layer is thickening. Second-mode instability waves are expected to grow inside the acoustic waveguide formed in between the wall and the location of the wall-normal gradient local maximum.

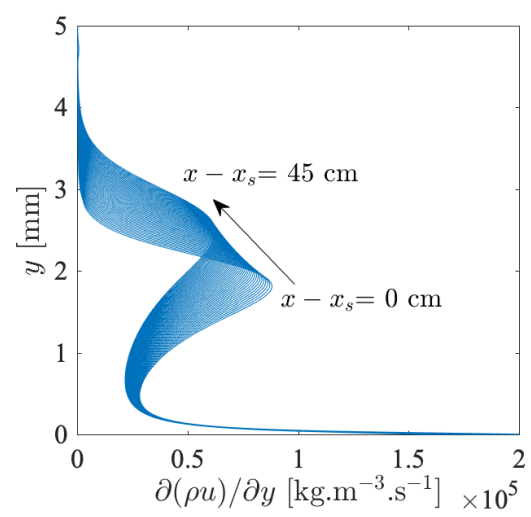

Fig. 7 Wall-normal gradient of streamwise momentum highlighting the impedance well structure.

\section{B. Steady excitation and growth of unstable modes over solid walls}

The solution is then perturbed by means of a wall-blowing source term. It is numerically imposed as a characteristic boundary condition covering a $0.5 \mathrm{~mm}$ long wall section centered on the $x-x_{s}=2.5 \mathrm{~cm}$ position. Specifically, it is enforced by the means of a characteristic decomposition as in Fiévet et al.[36], such that the wall pressure perturbation follows a sinusoidal function of amplitude $\rho c v_{0}$.

At first, a permanent harmonic forcing of amplitude $v_{0}=4.3 e^{-5} \times U_{\infty}=0.1 \mathrm{~m} \cdot \mathrm{s}^{-1}$ and frequencies $f \in$ $[180,240,260,280,300] \mathrm{kHz}$ is considered. Specifically, it is enforced by the means of a characteristic decomposition as in Fiévet et al. [36], such that the wall pressure perturbation follows a sinusoidal function of amplitude $\rho c v_{0}$. The activation of the source term results in a strong transient response from the flow with the appearance of a steady oblique shocklet attached to it, forming a $\sin ^{-1}\left(1 / M_{1}\right)$ angle with the wall. The pressure jump across it is on the order of a few pascal only.

Before proceeding with the activation of the UAC, the solid wall solution is validated by means of comparison with the LST. This time, the LST is based on the time-averaged solution of the unperturbed DNS rather than those of the precursor simulation. This will be the case for the remainder of the study for the sake of consistency. Figure 8 presents a comparison of $\mathrm{N}$-factor profiles obtained from the time-converged DNS and LST at all perturbation frequencies. Importantly, the LST N-factor plots have been rescaled in amplitude to account for the specific source location used with DNS. All profiles collapse reasonably well on the LST results given the simplification inherent to the LST formulation. The $260 \mathrm{kHz}$ case is further scrutinized by comparing wall-normal pressure perturbation amplitudes with the LST main unstable mode in Fig. 9 Again, the agreement between the hard wall DNS and the LST serves as an important validation of the SD flow solver and is a testament to its numerical accuracy in resolving the linear modal growth-rates over a broad spectral range.

Further, a numerical convergence study is presented in Fig. 10 The figure presents a close-up view of the $260 \mathrm{kHz}$ $\mathrm{N}$-factor plots towards the end of the domain, where it is at its highest value, for various cases. First, the grid resolution is assessed by running the same simulation on coarser (57 millions elements) and finer (560 millions elements) meshes. As is seen on the left plots, the discrepancies between all cases appear minimal, while the medium mesh (the one presented in Sec. III.Dp solution is virtually indistinguishable from the finest case considered.

Finally, a similar convergence study is performed on the forcing scheme. It is important to verify that the disturbance amplitude is small enough such its growth is governed by linear rates. The good comparison shown in Fig. 8 suggests that to be the case, but this sensitivity study is carried nonetheless. As with the mesh convergence plot, a decrease in source term amplitude has a marginal effect on the mode's growth which confirms that a $0.1 \mathrm{~m} . \mathrm{s}^{-1}$ forcing amplitude is suited to this study. As these sensitivity studies are performed on the most unstable mode for this particular configuration, it is considered to be a limiting case which validation implies all frequencies are adequately resolved. 


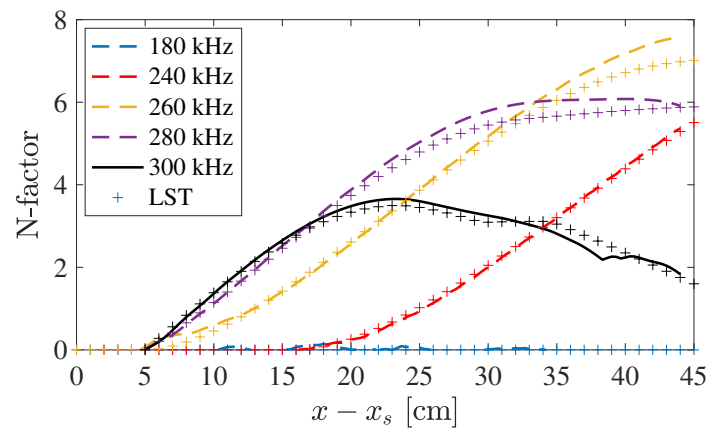

Fig. 8 Comparison of N-factor obtained from the DNS and LST for various source frequencies.
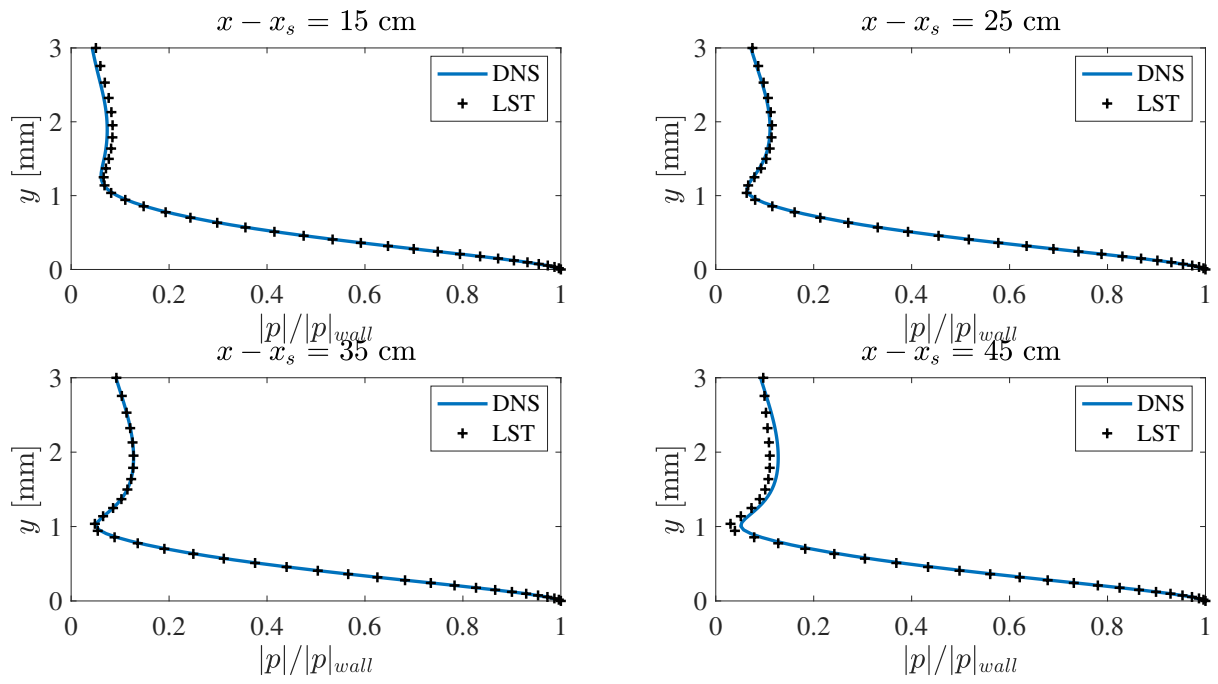

Fig. 9 Wall-normal pressure perturbations amplitudes at different streamwise locations for the LST and DNS solutions with solid wall boundary conditions under a $260 \mathrm{kHz}$ steady forcing.
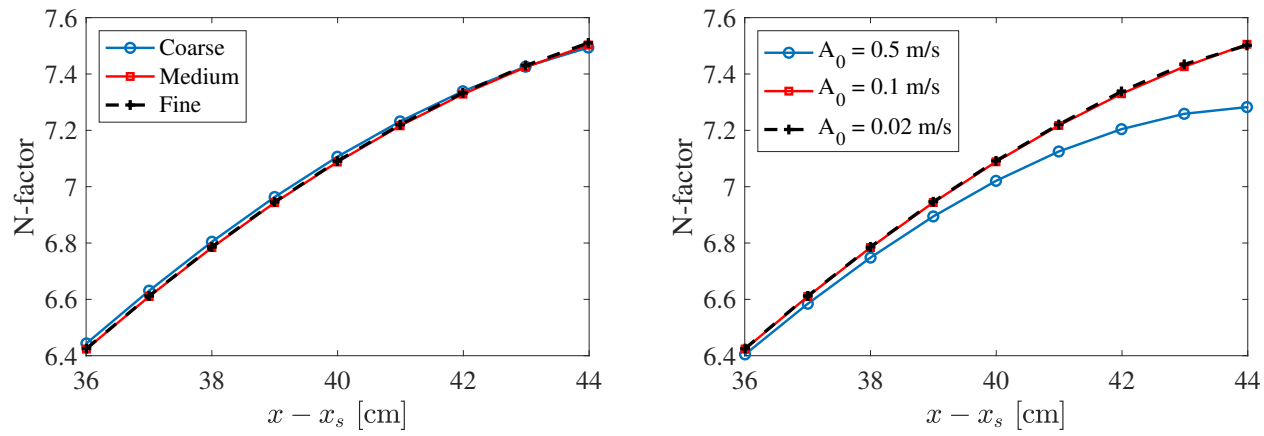

Fig. $10 \mathrm{~N}$-factor at the end of the domain for (left) different grid resolution and (right) source amplitudes.

\section{Dampening of unstable modes through UAC}

Having verified the accuracy of the solid wall simulation, the UAC (i.e. the TDIBC) is now activated with the hope of reducing, at first, the most-unstable mode growth. The simulation is initialized from the $260 \mathrm{kHz}$ forced steady-state solution. As the TDIBC activates at an instant defined as $t=0$, the abrupt change of wall-impedance triggers the appearance of transient pressure waves which radiate towards the far-field, before a steady-state is established. A strong 
and quick suppression of the instabilities is immediately observed along the wall, as seen in Fig. 11 where the wall pressure time-series is extracted at the outlet plane $\left(x-x_{s}=45 \mathrm{~cm}\right)$. A small time-lag can be noticed at start due to the finite travel time of information from $x-x_{s}=40$ (where the TDIBC ends) to the $x-x_{s}=45 \mathrm{~cm}$ probe location. It is followed by a rapid and strong damping of the harmonic perturbation. This stabilization is further quantified by comparing the wall pressure time-series and their power spectral density in Fig. 12 for the solid wall and TDIBC cases. The signals are extracted at $x-x_{s}=3.5$ and $40 \mathrm{~cm}$, which correspond to positions upstream and downstream of the TDIBC, respectively. The plot on the left shows a first important result: the TDIBC has no recursive effect on the upstream source. If it had been so, one would have raised questions on the pertinence of a direct comparison between the TDIBC and solid wall cases. As seen in the bottom right plots, the power spectral density peak value has been decreased by almost 6 orders of magnitude. This staggering decrease corresponds to a reduction in wall pressure oscillation amplitude by a factor 500, which is a testament to the UAC effectiveness in this idealized numerical environment.

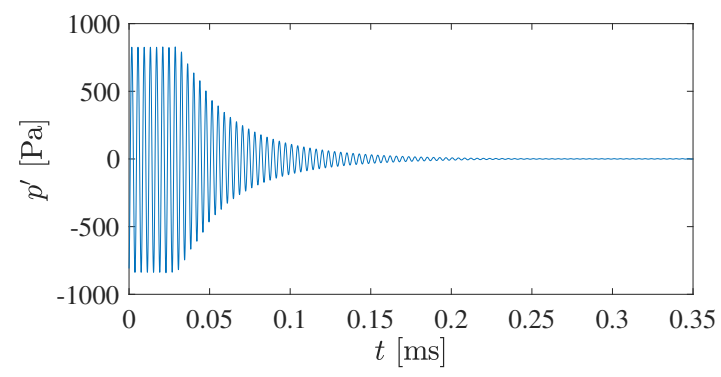

Fig. 11 Wall pressure time-series at $x-x_{s}=45 \mathrm{~cm}$ during the activation of the TDIBC (i.e. the UAC).
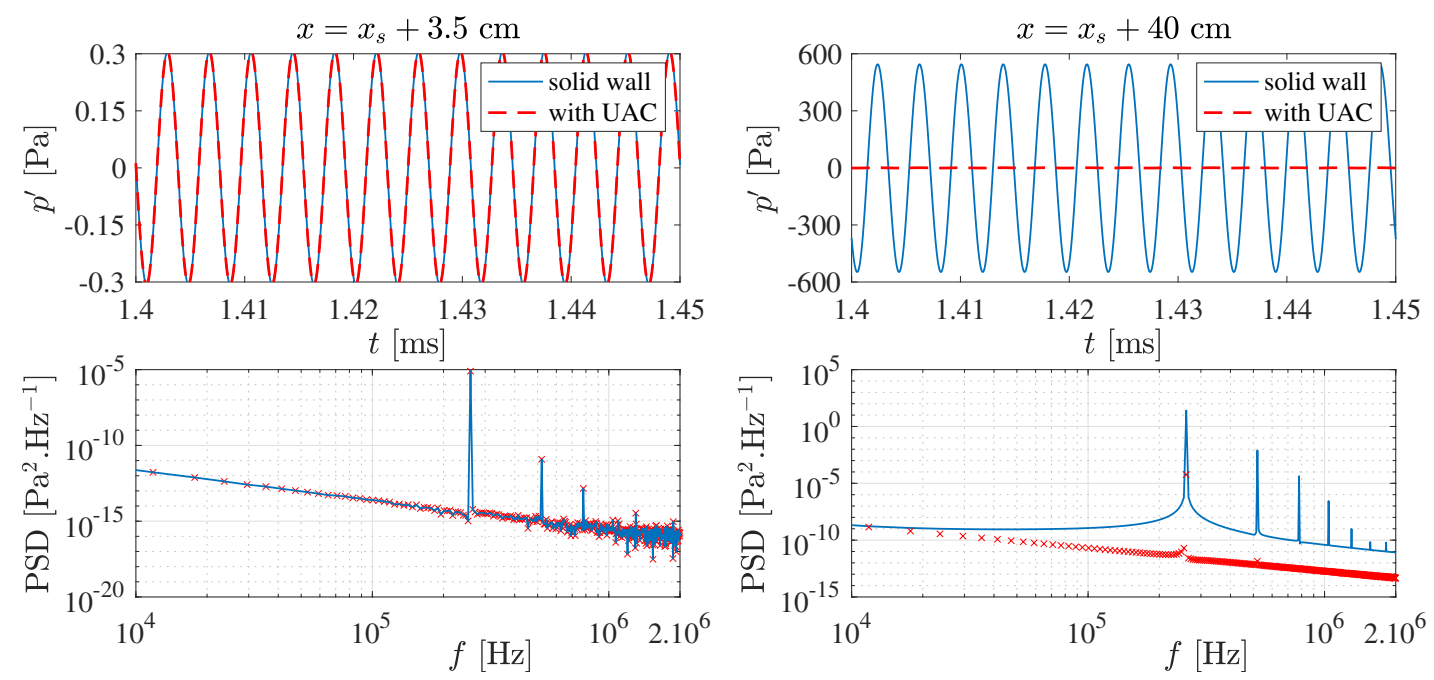

Fig. 12 (Top) Wall pressure time-signals at (Left) $x-x_{s}=3.5 \mathrm{~cm}$ and (Right) $x-x_{s}=40 \mathrm{~cm}$ alongside (bottom) their corresponding power spectral densities for the $260 \mathrm{kHz}$-excitation case over the solid and porous walls.

Similarly, the 2D contours presented in Fig. 13 illustrate how efficient the UAC is in constraining the pressure perturbations amplitudes. The contour at the top of Fig. 13 corresponds to the solid wall reference solution while the bottom one uses the TDIBC. They show a snapshot of the pressure fluctuations, obtained by subtracting from the instantaneous pressure field the average value calculated over one period of the steady excitation. A video file is provided as supplemental material. With the UAC, the amplitude of time-varying pressure fluctuations has decreased by a factor 500, to a level of only a few $\mathrm{Pa}$, which is consistent with the results obtained from the wall pressure probes.

A careful look at the bottom contour shows that some weak waves are emitted at $x-x_{s}=40 \mathrm{~cm}$, i.e at the end of the TDIBC wall. Actually, a steady train of weak Mach waves appears on the steady flow field, and builds up upstream of the wall impedance discontinuity. Their amplitude increases slightly with each period of the steady excitation. Typically, at $t=0.35 \mathrm{~ms}$, they modulate the steady pressure field by a maximum of $30 \mathrm{~Pa}$, i.e. less than $0.4 \%$ of the mean value. 


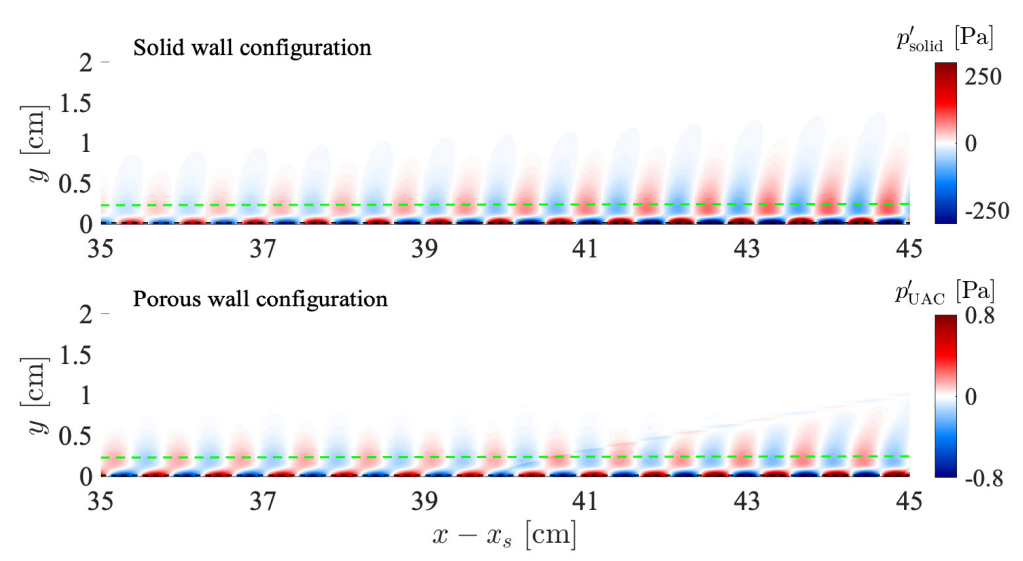

Fig. 13 Instantaneous contours of static pressure for the (top) solid and (bottom) porous wall configurations. Video file available as supplemental material.

Their impact on the stability properties of the boundary layer flow is therefore expected to be negligible. As these spurious pressure drifts are not time-varying, they are virtually invisible to the TDIBC. The use of relaxation term, as in typical non-reflecting pressure outflow boundary conditions, could help remove these spurious waves at the cost of affecting the TDIBC broadband response.

The LST code is then applied to the same base flow as in the previous section, but now with an impedance boundary condition at the wall. At each frequency, the complex value of the impedance is directly obtained from the reflection coefficient plotted in Fig. 11 The wall-normal profiles for the $260 \mathrm{kHz}$ case are compared to the DNS solution in Fig. 14 Even if the agreement is not as perfect as for the solid wall case (Fig. 9), the comparison is very satisfactory. This confirms that the train of Mach waves that appear on the steady mean flow in the DNS does not significantly impact the development of the instabilities. The plot for $x-x_{s}=45 \mathrm{~cm}$ shows the largest difference between the DNS and LST solutions. However, at this location, the LST solution is obtained with a solid wall boundary condition since the UAC ends at $x-x_{s}=40 \mathrm{~cm}$. It seems therefore that there is a history effect created by the UAC on the wall-normal evolution of the pressure perturbation, which cannot be captured by the LST approach.
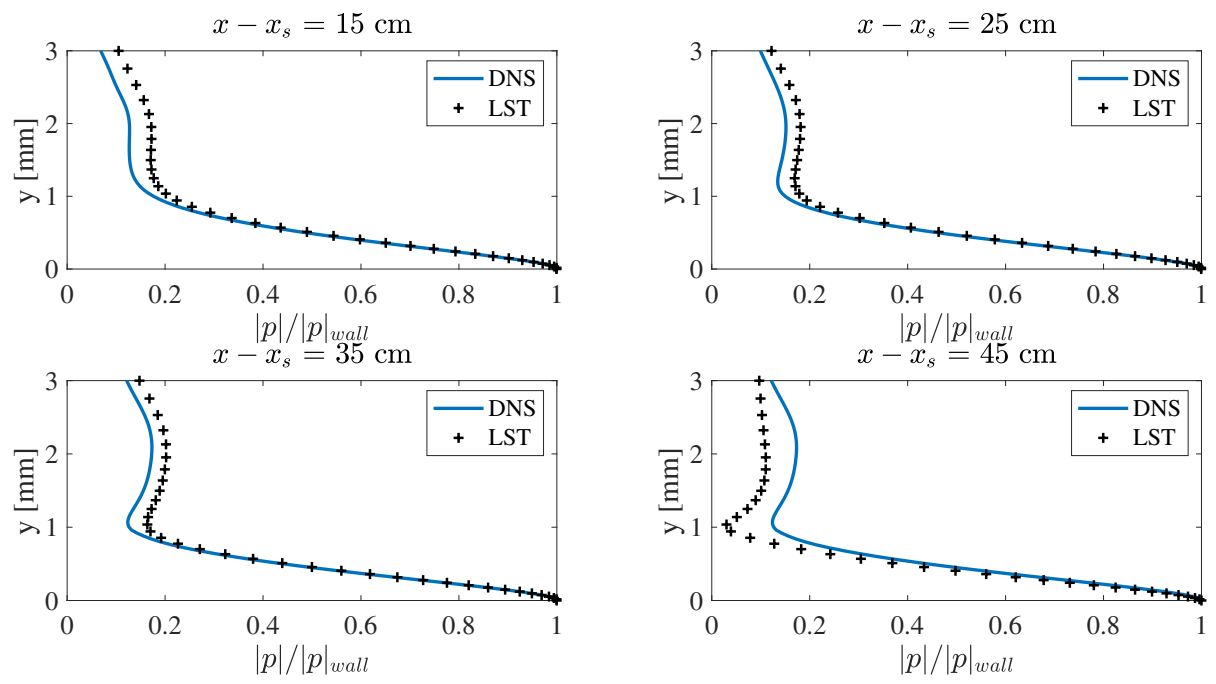

Fig. 14 Wall-normal pressure perturbations amplitudes at different streamwise locations for the LST and DNS solutions when using the UAC for the $260 \mathrm{kHz}$ mode.

A small parametric study was performed by changing the size of the coating in the DNS to cover the $x-x_{s} \in$ $[5,40],[15,40],[25,40],[35,40] \mathrm{cm}$ positions. $\Delta \mathrm{N}$-factor plots, defined as the difference between the UAC and solid 
wall N-factor plots, are presented in Fig. 15. The LST prediction is observed to be quite accurate, with a small overestimation of the UAC damping compared to the DNS solution. The differences between the LST and DNS are the smallest for the $5 \mathrm{~cm}$-long UAC (purple plots), and maximal for the $35 \mathrm{~cm}$-long UAC (blue plots). This effect is simply attributed to the differences between the DNS and LST building up over larger distances since their stabilization rates $\Delta \alpha_{i}$ appear to remain constant.

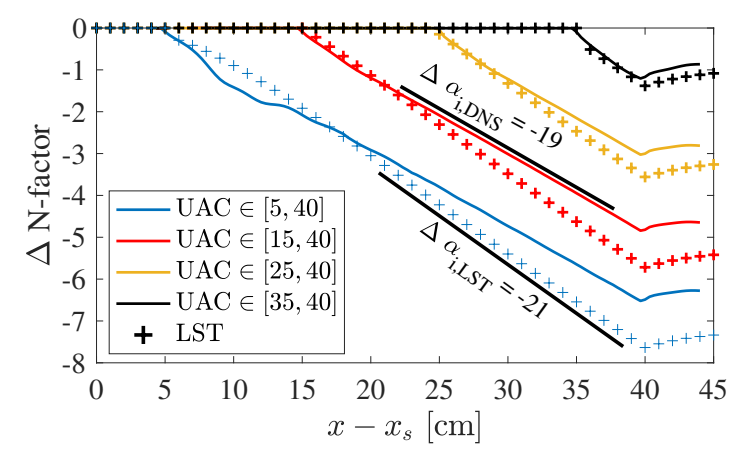

Fig. $15 \Delta \mathrm{N}$-factor profiles under $260 \mathrm{kHz}$ steady forcing for different UAC placements.

Next, the source frequency is varied to observe the UAC effect on other modes relevant to the present flow configuration. The $\Delta \mathrm{N}$-factor plots are presented in Fig. 16 for each $f$. All $\Delta \mathrm{N}$-factor profiles are plotted over their corresponding frequency's positive-growth regions. Note that the $180 \mathrm{kHz}$ case is not plotted as its growth region is downstream of the DNS computational domain. An additional $320 \mathrm{kHz}$ forcing case is run to better highlight the apparent steepening (i.e. better stabilization) of these profiles as frequencies increase. It is tempting to attribute this trend to numerical noise, as the lower growth-rate of higher modes decreases the measuring accuracy. However, power spectral density plots (not shown for the sake of conciseness) have shown that the excited mode is the largely dominant spectral component in all cases. Further, the discretization scheme of the TDIBC ensures sufficient resolution at these frequencies. Hence, the trend does suggest an increased absorption rate at higher frequencies for this particular UACas the $\mathrm{N}$-factor profile exhibit a sharper decrease rate as frequency increases. This result could not have been derived in view of the impedance law (Fig. 1) where the module of the reflection coefficient $\beta$ oscillates. This highlights the complexity of the wall impedance/unsteady grazing flow problem.

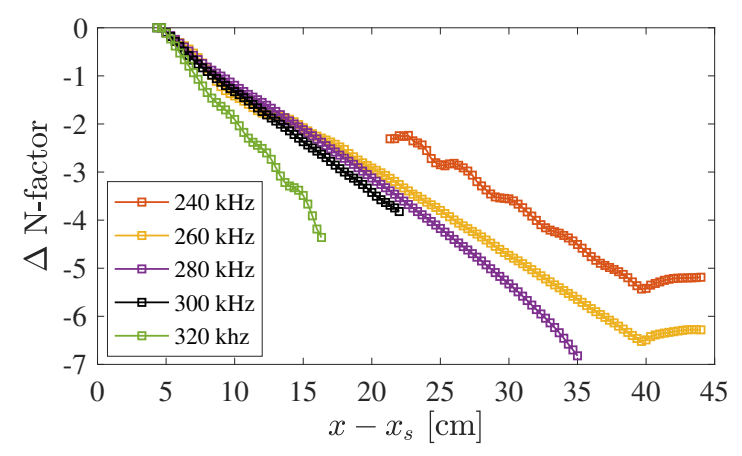

Fig. 16 Difference between the N-factor profiles at different excitation frequencies obtained from the solid and porous wall configurations. These profiles are only plotted over their respective solid-wall growth regions as shown in Fig. 4

Finally, another takeaway from Figs. 15 and 16 is that the passive control coating investigated in this work has a constant-rate impact in space, independently of its distance from the source or its frequency. Coincidentally, the most unstable frequency is known to vary and decrease along the streamwise direction as the boundary layer thickens: a spatially-inhomogeneous porous coating designed to damp the locally most-unstable mode would be an optimal flow-control material, as mentioned in [31]. 


\section{UAC stabilization capabilities of transient broadband noise}

This last set of simulations aims at ramping up the test complexity by considering a broadband forcing term. The permanent harmonic forcing is replaced by a single wavepacket. Its single-sided amplitude spectra resemblesa Gaussian curve centered on the main frequency of interest $(260 \mathrm{kHz})$, with a $10 \%$ relative amplitude at the $180 \mathrm{and} 340 \mathrm{kHz}$ bounds which roughly encompasses the unstable frequencies over the DNS domain. The peak amplitude remains equal to $0.1 \mathrm{~m} . \mathrm{s}^{-1}$. The simulation is initialized with the steady-state, inert solution presented in Sec. III.A The source is activated at $t=0$ for a period of time corresponding to six $260 \mathrm{kHz}$-periods, i.e. $23.1 \mu \mathrm{s}$. Afterwards, the blowing condition is replaced by an isothermal fully reflective boundary condition. As before, one simulation activates the TDIBC while the other does not. Snapshots of pressure perturbations for the former case are shown in Fig. 17] ordered in time from top to bottom. They show the wavepacket evolution as it travels downstream, all along the DNS domain. As the wavepacket grazes over the UAC, its amplitude and shape is modified, but its spatial structure remains quite similar to the one observed in the permanent forcing case (see Fig. 13). These contours also shed some light on the oblique Mach waves that appear at each change of the wall boundary condition. The oblique shocklet created at $x-x_{s}=2.5 \mathrm{~cm}$ by the wall-blowing forcing is clearly visible; its amplitude decreases over time. At the end of the TDIBC wall $\left(x_{s}=40\right.$ $\mathrm{cm}$ ), some weak oblique Mach waves can be observed from the start, though of amplitude of $0.02 \mathrm{~Pa}$. As discussed in the previous section, their amplitude increases slightly whenever a pressure perturbation crosses the UAC-to-solid wall discontinuity. These spurious imprints grow in amplitude as the wavepacket crosses that point. As these artefacts are static and of very low amplitude (about $0.1 \mathrm{~Pa}$ after the wavepacket passage) we do not believe they impact the following analysis. However, they certainly warrant some concern on the stability of high-order TDIBC in combination with discontinuous spectral methods.

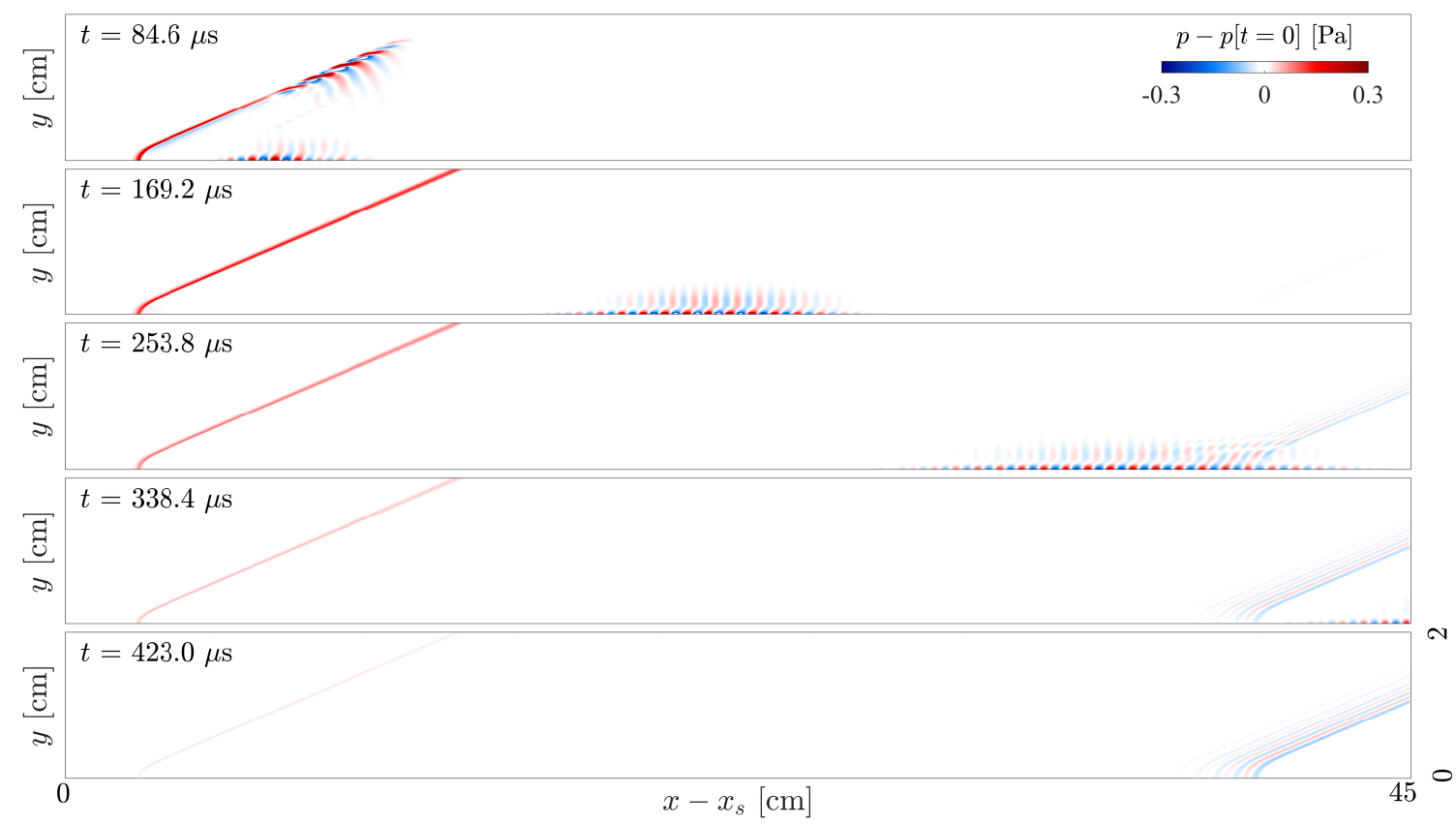

Fig. 17 Contours of static pressure showing the wavepacket propagation in time (increasing from top to bottom). Note that the coordinate system is non-orthonormal.

The UAC and solid wall solutions are now compared in Figure 18 which presents some wall pressure probes signals at various location for both cases. All signals are normalized by the wavepacket's envelop maximum, as indicated in each subplot. Unsurprisingly, the UAC is very efficient at containing the wavepacket growth within its initial bounds. On the other hand, the solid wall wavepacket exponential growth results in an envelop peak amplitude well over 100 Pascal.

Strikingly, both cases experience severe dispersion as the initial wavepacket widens. Further, the solid wall wavepacket has maintained its Gaussian shape while the one grazing over the TDIBC exhibits a skewer profile. This means the boundary layer dispersion properties have been affected by the coating. Finally, it appears that the wavepacket has convected at a higher speed than in the solid wall case: it reaches the $x-x_{s}$ location half a millisecond earlier. 

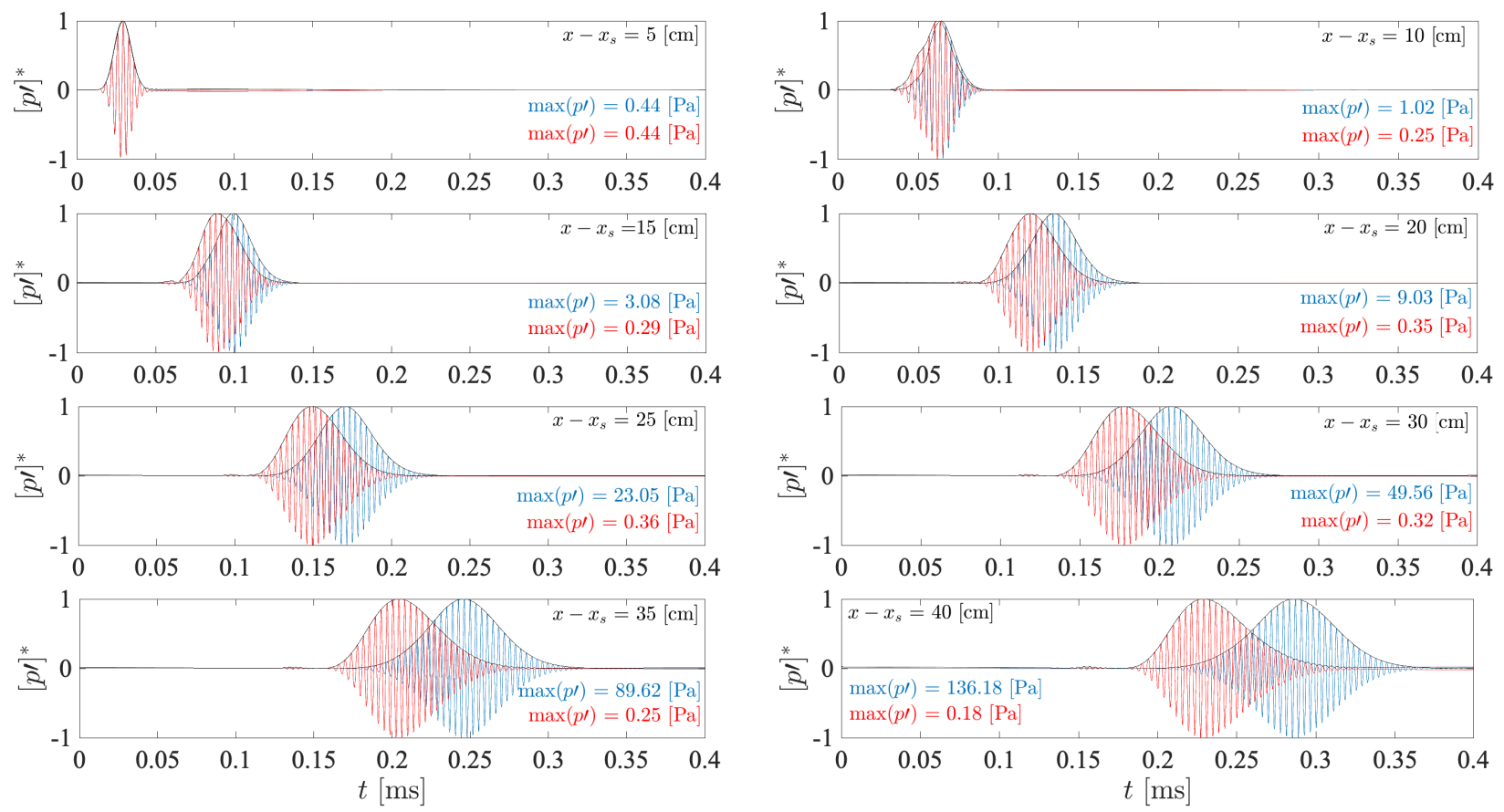

Fig. 18 Wall pressure time-series at various streamwise location under wavepacket excitation with (blue) solid wall and (red) UAC.

This result is further illustrated by the space-time diagram of the peak envelop location shown in Fig. 19. Overall, the wavepacket group velocity amounts to 59 and $79 \%$ of the bulk velocity in the solid and porous wall cases, respectively, and stays remarkably constant over the $[5,45] \mathrm{cm}$ region.

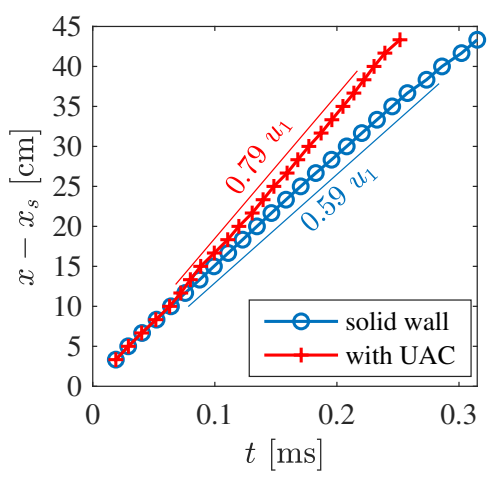

Fig. 19 Location of the wavepacket's envelope peak value over time for the (blue) solid wall and (red) UAC.

In order to determine the cause of such drastic modification of the wavepacket's properties, the wall pressure timeseries at the $x-x_{s}=40 \mathrm{~cm}$ location are further scrutinized. Their corresponding single-sided amplitude spectra (SSAS) are presented in Fig. 20 for both cases. First, it clearly appears that the solid wall SSAS has departed from its original Gaussian-like spectral distribution, with a sensible increase in high-frequencies amplitudes. This is evidently caused by the unequal growth of modes of frequency superior $260 \mathrm{kHz}$ in this region. Second, the porous wall SSAS exhibits is instead strongly skewed towards the lower frequencies. Again, this result is expected in view of the analysis presented in Fig. 16 which showed higher damping rates for the higher-frequencies. As such, the spectral balance has shifted towards the lower frequencies. As a result, the wavepacket's group velocity is increasingly determined by the 
phase velocities of higher (resp. lower) frequencies in the solid (resp. porous) wall case.

Finally, the phase velocities in the $[180,320] \mathrm{kHz}$ spectral range are evaluated from the DNS and the LST as well. They are shown in Fig. 21] where, for better clarity, they have been averaged over the $x-x_{s} \in$ [540] region. The following comment are made:

1) The lowest number of element-per-wavelength in this frequency-velocity space is around 12 (a $320 \mathrm{kHz}$ wave traveling at $0.75 u_{1}$ has a wavelength of $6 \mathrm{~mm}$ ). This corresponds to 48 solution points per wavelength as a fourth-order SD scheme is used. As explained in [38], less than $1 \%$ of the wave energy would be dissipated after 1 million iterations (see Table 7 of this paper) which confirms that the DNS is properly resolved.

2) The LST and DNS are in good agreement in the spectral region which dominates the flow (that is between 220 and $280 \mathrm{kHz}$ as seen in Fig. 20p, and departs outside of the bound of the initial Gaussian distribution. As per point 1 , this is not caused by a supposedly lack of spatial resolution. Instead, this discrepancies is likely due to evaluating phase velocities from quasi-null timeseries.

3) Both the DNS and LST show that the phase velocities change between the solid and porous wall cases. This means the medium dispersive properties have been somewhat affected by the coating.

4) The lower frequencies have a higher phase velocity, with or without UAC, but the use of the porous coating does decrease this difference.

To conclude, the increased dampening of the slow-propagating high-lying frequencies relative to the fast-propagating lower ones has shifted the spectral balance. As a result, the group velocity has increased. Interestingly, the DNS phase velocities plots for the solid and porous wall cases intersect at the flow bulk velocity $u_{1}$ isoline. To the best understanding of the authors, this might very well be a coincidence and no satisfactory explanation is found.

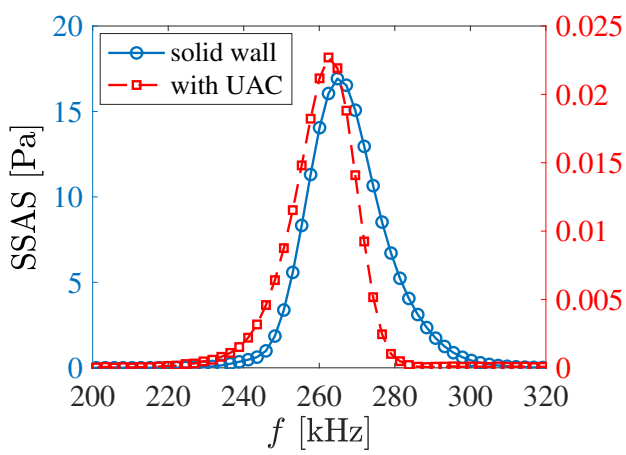

Fig. 20 Single-sided amplitude spectra of the normalized wall pressure time-series $\left[p^{\prime}\right]^{*}$ at $x-x_{s}=\mathbf{4 0} \mathrm{cm}$ for the (blue) solid wall and (red) UAC.

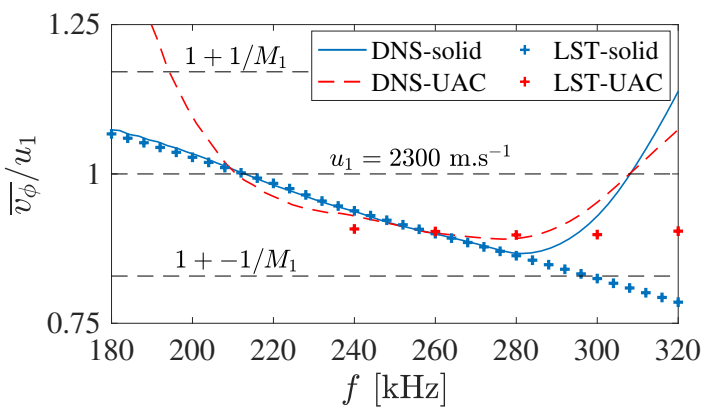

Fig. 21 Phase velocities averaged over the region $x-x_{s} \in[5,40] \mathrm{cm}$ for the DNS and the LST.

\section{Summary and conclusions}

This numerical work investigates the second-mode stabilizing capacities of UAC in hypersonic boundary layers. It combines both linear stability theory and direct numerical simulations to study a realistic configuration inspired by the 
experiment of Wagner et al. [19]. These high-fidelity simulations use a high-order spectral difference method, which is a novelty in the simulation of laminar-turbulent transition. This discontinuous scheme possesses a spectral-like accuracy which allows for a detailed resolution of a wide range of wavenumbers: it is a prerequisite to properly capture the physical growth of unstable modes in a nonlinear framework. Further, the SD method retains a high-order of accuracy on unstructured grid thanks to its compact formalism. This would enable similar studies on complex geometries in the near future. A time-domain impedance boundary condition, as implemented in [34], resolves the effect of the UAC on the flow. Its coupling with the Navier-Stokes solver occurs through a reflectivity condition imposed in characteristic-space. The TDIBC is represented, following the method of [33], by a sum of weighted acoustic poles which are calibrated to fit a target impedance law corresponding to the coating of interest. As a result, it is tailored to provide a broadband-accurate representation of the coating's impedance condition within the time-domain. The conjunction of both types of boundary condition and spectral methods has resulted in the development of a high-order numerical flow solver well-equipped to study the underlying physics of hypersonic boundary-layer transition and eventually design optimal UAC.

Many simulations have been performed in this study, they are divided into three kinds described as follows.

- Unperturbed simulations have verified the passivity of the TDIBC: that is, in absence of any wall pressure fluctuation, the steady-state solution is unchanged from a solid wall (UAC-less) simulation.

- Under permanent harmonic forcing, the modal growths compare well with Linear Stability Theory, with and without UAC. This shows that 1. the SD method is able to resolve the growth of unstable modes and 2. the LST can correctly capture the effect of a complex wall impedance boundary condition on said growth. These simulations also highlight the stabilizing capabilities that a well-designed UAC can achieve in an ideal numerical environment. Further, the absorption rate of the said coating was shown to be constant over the material and only depend on frequency, albeit not simply as the impedance law would suggest.

- Under intermittent broadband forcing (in the form of a single pressure wavepacket), the UAC was shown to affect the boundary layer dispersive properties and spectral distribution due to its uneven absorptive properties. As some frequencies were better canceled than others, the spectral balance shifted and the wavepacket group velocity changed. The LST provided good estimate of phase velocities and modal growth rates.

These results show that the design and optimization of complex UAC, defined by many acoustic poles, can be achieved numerically by carrying out high-order numerical simulations as done in this study. It was also shown that LST can, despite their simplified approach, provide a surprisingly good estimate of the effect of UAC on the flow. Finally the observation of a buildup in static pressure perturbations on the DNS mean field (as low-amplitude it may be) raises the question of numerical stability. As these spurious oscillations are not of time-varying nature, they are virtually invisible to the TDIBC which explains its resilience to this numerical artefact. Overall, this issue requires further scrutiny.

\section{Appendix}

The table below presents all the scalars used to discretize the TDIBC matching the broadband profile shown in Fig. 1

\section{Funding Sources}

The first author is funded by the STAE foundation (Science and Technology for Aeronautics and Space), grant number CDT-R050-L00-T00. This work is part of the STAE-funded 3C2T project (control of compressible, transitional and turbulent boundary layer) involving researchers from ONERA, CERFACS and the Institute of Fluid Mechanics in Toulouse (IMFT) under the supervision of Christophe Airiau (IMFT).

\section{Acknowledgments}

The authors thank Loïc Jecker and José Cardesa for their kind help in configuring and handling the precursor simulation using the code CEDRE.

\section{References}

[1] Reed, H., Kimmel, R., Schneider, S., and Arnal, D., "Drag prediction and transition in hypersonic flow, AIAA Paper 1997-1818," AIAA 28th Fluid Dynamics Conference, 1997. https://doi.org/10.2514/6.1997-1818 


\begin{tabular}{|c|c|c|}
\hline \hline Scalars & $\beta_{\infty}=8.187879 e^{-1}$ & $\tau=5.877685 e^{-6} \mathrm{~s}$ \\
\hline Poles & \multicolumn{2}{|c|}{ Functions weights } \\
\hline$\left(\tilde{s}_{n},-\tilde{\xi}_{k}\right)_{n, k}\left({\left.\mathrm{rad} . \mathrm{s}^{-1}\right)}^{-1.256637 \mathrm{e}+3}\right.$ & $\left(\tilde{r}_{1, n},-\tilde{\mu}_{1, k}\right)_{n, k}\left(\mathrm{rad} . \mathrm{s}^{-1}\right)$ & $\left(\tilde{r}_{2, n},-\tilde{\mu}_{2, k}\right)_{n, k}\left(\mathrm{rad} . \mathrm{s}^{-1}\right)$ \\
\hline$-2.148819 \mathrm{e}+4$ & $5.660893 \mathrm{e}+0$ & $4.277775 \mathrm{e}+0$ \\
\hline$-3.674429 \mathrm{e}+5$ & $6.807834 \mathrm{e}+2$ & $9.184530 \mathrm{e}+2$ \\
\hline$-6.283185 \mathrm{e}+6$ & $3.589210 \mathrm{e}+4$ & $1.261821 \mathrm{e}+4$ \\
\hline$(-1.974050 \mathrm{e}+5,3.1685357 \mathrm{e}+5)$ & $-1.331916 \mathrm{e}+6$ & $-4.117427 \mathrm{e}+4$ \\
\hline$(-3.544161 \mathrm{e}+2,1.234667 \mathrm{e}+6)$ & $(-5.737098 \mathrm{e}+4,-8.382613 \mathrm{e}+3)$ & $(1.115722 \mathrm{e}+4,-2.097452 \mathrm{e}+4)$ \\
\hline$(-4.614602 \mathrm{e}+5,2.198411 \mathrm{e}+6)$ & $(-5.601881 \mathrm{e}+4,-1.478211 \mathrm{e}+4)$ & $(-4.509185 \mathrm{e}+3,-2.663290 \mathrm{e}+3)$ \\
\hline$(-5.482225 \mathrm{e}+5,3.181595 \mathrm{e}+6)$ & $(-6.548491 \mathrm{e}+4,-1.802480 \mathrm{e}+4)$ & $(-2.827210 \mathrm{e}+3,-2.504131 \mathrm{e}+2)$ \\
\hline$(-6.231411 \mathrm{e}+5,4.176283 \mathrm{e}+0)$ & $(-8.044434 \mathrm{e}+4,-1.886116 \mathrm{e}+4)$ & $(-1.356928 \mathrm{e}+3,2.770425 \mathrm{e}+2)$ \\
\hline$(-6.900477 \mathrm{e}+5,5.178806 \mathrm{e}+6)$ & $(-1.101268 \mathrm{e}+5,-1.467016 \mathrm{e}+4)$ & $(-3.609999 \mathrm{e}+2,-1.731994 \mathrm{e}+2)$ \\
\hline$(-7.510679 \mathrm{e}+5,6.187110 \mathrm{e}+6)$ & $(-2.339126 \mathrm{e}+5,-2.490394 \mathrm{e}+4)$ & $(1.160141 \mathrm{e}+3,-2.148801 \mathrm{e}+3)$ \\
\hline$(-8.075265 \mathrm{e}+5,7.199905 \mathrm{e}+6)$ & $(-5.691639 \mathrm{e}+4,-5.317827 \mathrm{e}+5)$ & $(6.532230 \mathrm{e}+3,3.590720 \mathrm{e}+3)$ \\
\hline
\end{tabular}

Table 2 Scalar parameters defining the oscillo-diffusive representation of the UAC presented in Fig. 1.

[2] Zhong, X., and Wang, X., "Direct numerical simulation on the receptivity, instability, and transition of hypersonic boundary layers," Annual Review of Fluid Mechanics, Vol. 44, 2012, pp. 527-561. https://doi.org/10.1146/annurev-fluid-120710-101208

[3] Fedorov, A., "Transition and stability of high-speed boundary layers," Annual Review of Fluid Mechanics, Vol. 43, 2011, pp. 79-95. https://doi.org/10.1146/annurev-fluid-122109-160750

[4] Malik, M., Zang, T., and Bushnell, D., "Boundary layer transition in hypersonic flows, AIAA Paper 90-5232," 2nd AIAA International Aerospace Planes Conference, Orlando, 1990. https://doi.org/10.2514/6.1990-5232.

[5] Mack, L., "Review of linear compressible stability theory," Stability of Time Dependent and Spatially Varying Flows, edited by D. Dwoyer and M. Hussaini, Springer, 1987, pp. 164-187. https://doi.org/10.1007/978-1-4612-4724-1_9

[6] Morkovin, M. V., "Transition at hypersonic speeds,” Technical Report ICASE, NASA Contractor Report $178315,1987$.

[7] Mack, L., "On the inviscid acoustic-mode instability of supersonic shear flows. Part 1: two-dimensional waves," Theoretical and Computational Fluid Dynamics, Vol. 2, No. 2, 1990, pp. 97-123. https://doi.org/10.1007/BF00272137.

[8] Egorov, I. V., Fedorov, A. V., and Soudakov, V. G., "Receptivity of a hypersonic boundary layer over a flat plate with a porous coating," Journal of Fluid Mechanics, Vol. 601, 2008, p. 165-187. https://doi.org/10.1017/S0022112008000669.

[9] Li, X., Fu, D., and Ma, Y., “Direct Numerical Simulation of Hypersonic Boundary Layer Transition over a Blunt Cone,” AIAA Journal, Vol. 46, No. 11, 2008, pp. 2899-2913.

[10] Knisely, C. P., and Zhong, X., "Sound radiation by supersonic unstable modes in hypersonic blunt cone boundary layers. I. Linear stability theory," Physics of Fluids, Vol. 31, No. 2, 2019, p. 024103. https://doi.org/10.1063/1.5055761

[11] Knisely, C. P., and Zhong, X., "Sound radiation by supersonic unstable modes in hypersonic blunt cone boundary layers. II. Direct numerical simulation," Physics of Fluids, Vol. 31, No. 2, 2019, p. 024104. https://doi.org/10.1063/1.5077007

[12] Xu, J., Liu, J., Mughal, S., Yu, P., and Bai, J., "Secondary instability of Mack mode disturbances in hypersonic boundary layers over micro-porous surface," Physics of Fluids, Vol. 32, No. 4, 2020, p. 044105. https://doi.org/10.1063/5.0001914

[13] Kuehl, J. J., “Thermoacoustic Interpretation of Second-Mode Instability,” AIAA Journal, Vol. 56, No. 9, 2018 , pp. 3585-3592. https://doi.org/10.2514/1.J057015

[14] Mack, L., "Effect of cooling on boundary-layer stability at Mach number 3," Instabilities and Turbulence in Engineering Flows, edited by D. Ashpis, T. Gatski, and R. Hirsh, Springer, 1993, pp. 175-188. https://doi.org/10.1007/978-94-011-1743-2_9. 
[15] Fedorov, A., Malmuth, N., Rasheed, A., and Hornung, H., "Stabilization of hypersonic boundary layers by porous coatings," AIAA Journal, Vol. 39, No. 4, 2001, pp. 605-610. https://doi.org/10.2514/2.1382.

[16] Rasheed, A., Hornung, H., Fedorov, A., and Malmuth, N., "Experiments on passive hypervelocity boundary-layer control using an ultrasonically absorptive surface," AIAA Journal, Vol. 40, No. 3, 2002, pp. 481-489. https://doi.org/10.2514/2.1671

[17] Fedorov, A., Shiplyuk, A., Maslov, A., Burov, E., and Malmuth, N., "Stabilization of a hypersonic boundary layer using an ultrasonically absorptive coating," Journal of Fluid Mechanics, Vol. 479, 2003, pp. 99-124. https://doi.org/10.1017/ S0022112002003440

[18] Bountin, D., Chimitov, T., Maslov, A., Novikov, A., Egorov, I., Fedorov, A., and Utyuzhnikov, S., "Stabilization of a Hypersonic Boundary Layer Using a Wavy Surface," AIAA Journal, Vol. 51, No. 5, 2013, pp. 1203-1210.

[19] Wagner, A., Kuhn, M., Martinez Schramm, J., and Hannemann, K., "Experiments on passive hypersonic boundary layer control using ultrasonically absorptive carbon-carbon material with random microstructure," Experiments in Fluids, Vol. 54, 2013, p. 1606. https://doi.org/10.1007/s00348-013-1606-3

[20] Wagner, A., Hannemann, K., and Kuhn, M., "Ultrasonic absorption characteristics of porous carbon-carbon ceramics with random microstructure for passive hypersonic boundary layer transition control," Experiments in Fluids, Vol. 55, 2014 , p. 1750. https://doi.org/10.1007/s00348-014-1750-4

[21] Wartemann, V., Wagner, A., Eggers, T., and Hannemann, K., "Passive hypersonic boundary layer control by means of ultrasonically absorptive carbon-carbon ceramics: Investigations of different boundary conditions, AIAA Paper 20153577," 20th AIAA International Space Planes and Hypersonic Systems and Technologies Conference, Glasgow, 2015. https://doi.org/10.2514/6.2015-3577

[22] Brès, G., Inkman, M., Colonius, T., and Fedorov, A., "Second-mode attenuation and cancellation by porous coatings in a high-speed boundary layer," Journal of Fluid Mechanics, Vol. 726, 2013, pp. 312-337. https://doi.org/10.1017/jfm.2013.206

[23] Sandham, N. D., and Lüdeke, H., "Numerical Study of Mach 6 Boundary-Layer Stabilization by Means of a Porous Surface," AIAA Journal, Vol. 47, No. 9, 2009, pp. 2243-2252. https://doi.org/10.2514/1.43388

[24] De Tullio, N., and Sandham, N. D., "Direct numerical simulation of breakdown to turbulence in a Mach 6 boundary layer over a porous surface," Physics of Fluids, Vol. 22, No. 9, 2010, p. 094105. https://doi.org/10.1063/1.3481147.

[25] Hader, C., Brehm, C., and Fasel, H. F., "Numerical Investigation of transition delay for various controlled breakdown scenarios in a Mach 6 Boundary Layer using porous walls, AIAA Paper 2014-2500," 7th AIAA Theoretical Fluid Mechanics Conference, 2014. https://doi.org/10.2514/6.2020-2061.

[26] Zhao, R., Wen, C. Y., Long, T. H., Tian, X. D., Zhou, L., and Wu, Y., "Spatial Direct Numerical Simulation of the Hypersonic Boundary-Layer Stabilization Using Porous Coatings," AIAA Journal, Vol. 57, No. 11, 2019, pp. 5061-5065. https://doi.org/10.2514/1.J058467

[27] Tritarelli, R. C., Lele, S. K., and Fedorov, A., "Stabilization of a hypersonic boundary layer using a felt-metal porous coating," Journal of Fluid Mechanics, Vol. 769, 2015, p. 729-739. https://doi.org/10.1017/jfm.2015.156

[28] Zhao, R., Liu, T., Wen, C., Zhu, J., and Cheng, L., "Theoretical modeling and optimization of porous coating for hypersonic laminar flow control,” AIAA Journal, Vol. 56, No. 8, 2018, pp. 2942-2946. https://doi.org/10.2514/1.J057272

[29] Sousa, V., Patel, D., Chapelier, J.-B., Wagner, A., and Scalo, C., "Numerical investigation of second mode attenuation over carbon/carbon surfaces on a sharp slender cone, AIAA Paper 2018-0350," 2018 AIAA Aerospace Sciences Meeting, Kissimmee, 2018. https://doi.org/10.2514/6.2018-0350

[30] Sousa, V. C. B., Patel, D., Chapelier, J.-B., Wartemann, V., Wagner, A., and Scalo, C., "Numerical Investigation of Second-Mode Attenuation over Carbon/Carbon Porous Surfaces," Journal of Spacecraft and Rockets, Vol. 56, No. 2, 2019 , pp. 319-332. https://doi.org/10.2514/1.A34294

[31] Tian, X., Zhao, R., Long, T., and Wen, C. Y., "Reverse Design of Ultrasonic Absorptive Coating for the Stabilization of Mack Modes," AIAA Journal, Vol. 57, No. 6, 2019, pp. 2264-2269. https://doi.org/10.2514/1.J058105

[32] Monteghetti, F., Matignon, D., Piot, E., and Pascal, L., "Design of broadband time-domain impedance boundary conditions using the oscillatory-diffusive representation of acoustical models," The Journal of the Acoustical Society of America, Vol. 140, No. 3, 2016, pp. 1663-1674. https://doi.org/10.1121/1.4962277 
[33] Monteghetti, F., Matignon, D., and Piot, E., "Energy analysis and discretization of nonlinear impedance boundary conditions for the time-domain linearized Euler equations," Journal of Computational Physics, Vol. 375, 2018, pp. 393 - 426. https://doi.org/https://doi.org/10.1016/j.jcp.2018.08.037

[34] Fiévet, R., Deniau, H., and Piot, E., "Strong compact formalism for characteristic boundary conditions with discontinuous spectral methods," Journal of Computational Physics, Vol. 408, 2020, p. 109276. https://doi.org/https://doi.org/10.1016/j.jcp. 2020.109276

[35] Poinsot, T. J., and Lele, S. K., "Boundary Conditions for Direct Simulations of Compressible Viscous Flows," Journal of Computational Physics, Vol. 101, 1992, pp. 104-129.

[36] Fiévet, R., Deniau, H., Brazier, J.-P., and Piot, E., "Numerical Study of Hypersonic Boundary-Layer Transition Delay through Second-Mode Absorption, AIAA Paper 2020-2061,” AIAA Scitech Forum, 2020. https://doi.org/10.2514/6.2020-2061

[37] Cardesa, J. I., Hascoet, L., and Airiau, C., "Adjoint computations by algorithmic differentiation of a parallel solver for time-dependent PDEs,", 2019.

[38] Vanharen, J., Puigt, G., Vasseur, X., Boussuge, J.-F., and Sagaut, P., "Revisiting the spectral analysis for high-order spectral discontinuous methods," Journal of Computational Physics, Vol. 337, 2017, pp. 379 - 402. https://doi.org/https: //doi.org/10.1016/j.jcp.2017.02.043

[39] Lele, S. K., "Compact finite difference schemes with spectral-like resolution,” Journal of Computational Physics, Vol. 103, No. 1, 1992, pp. 16 - 42. https://doi.org/https://doi.org/10.1016/0021-9991(92)90324-R.

[40] Bogey, C., and Bailly, C., "A family of low dispersive and low dissipative explicit schemes for flow and noise computations," Journal of Computational Physics, Vol. 194, No. 1, 2004, pp. 194 - 214. https://doi.org/https://doi.org/10.1016/j.jcp.2003.09.003

[41] Cassagne, A., Boussuge, J.-F., and Puigt, G., "High-order Method for a New Generation of Large Eddy Simulation Solver," Technical report, PRACE, 2015.

[42] Hamri, W., "Evaluation of the JAGUAR solver with benchmark test cases," Rapport technique cerfacs, 2015.

[43] Scherrer, D., Dessornes, O., Ferrier, M., Vincent-Randonnier, A., Sabel'nikov, V., and Moule, Y., "Research on Supersonic Combustion and Scramjet Combustors at ONERA," AerospaceLab Journal, , No. 11, 2016.

[44] Refloch, A., Courbet, B., Murrone, A., Villedieu, P., Laurent, C., Gilbank, P., Troyes, J., Tessé, L., Chaineray, G., Dargaud, J.-B., Quémerais, E., and Vuillot, F., “CEDRE Software,” AerospaceLab Journal, , No. 2, 2011.

[45] Brazier, J.-P., Perraud, J., and Couzi, J., "Transition prediction on Reentry-F trajectory with PSE at chemical equilibrium," International Conference on Flight Vehicles, Aerothermodynamics and Re-entry Missions \& Engineering, Monopoli, 2019.

[46] Toro, E. F., Spruce, M., and Speares, W., "Restoration of the contact surface in the HLL-Riemann solver," Shock Waves, Vol. 4, No. 1, 1994, pp. 25-34. https://doi.org/10.1007/BF01414629

[47] Esquieu, S., Benitez, E., Schneider, S. P., and Brazier, J.-P., "Flow and Stability Analysis of a Hypersonic Boundary Layer over an Axisymmetric Cone Cylinder Flare Configuration, AIAA Paper 2019-2115," AIAA Scitech Forum, 2019. https://doi.org/10.2514/6.2019-2115 